\title{
A configurational force for adaptive re-meshing of gradient-enhanced poromechanics problems with history-dependent variables
}

\author{
SeonHong $\mathrm{Na}^{\mathrm{a}}$, Eric C. Bryant ${ }^{\mathrm{b}}$, WaiChing $\mathrm{Sun}^{\mathrm{c}, *}$ \\ ${ }^{a}$ Department of Civil Engineering, McMaster University, Hamilton, ON, Canada \\ ${ }^{\mathrm{b}}$ Department of Civil Engineering and Engineering Mechanics, Columbia University, New York, NY, USA \\ ${ }^{\mathrm{c}}$ Department of Civil Engineering and Engineering Mechanics, Columbia University, 614 SW Mudd, Mail Code: 4709, New York, NY 10027, USA
}

Received 22 March 2019; received in revised form 5 July 2019; accepted 30 July 2019

Available online xxxx

\begin{abstract}
We introduce a mesh-adaption framework that employs a multi-physical configurational force and Lie algebra to capture multiphysical responses of fluid-infiltrating geological materials while maintaining the efficiency of the computational models. To resolve sharp gradients of both displacement and pore pressure, we introduce an energy-estimate-free re-meshing criterion by extending the configurational force theory to consider the energy dissipation due to the fluid diffusion and the gradient-dependent plastic flow. To establish new equilibria after remeshing, the local tensorial history-dependent variables at the integration points are first decomposed into spectral forms. Then, the principal values and directions are projected onto smooth fields interpolated by the basis function of the finite element space via the Lie-algebra mapping. Our numerical results indicate that this Lie algebra operator in general leads to a new trial state closer to the equilibrium than the ones obtained from the tensor component mapping approach. A new configurational force for dissipative fluid-infiltrating porous materials that exhibit gradient-dependent plastic flow is introduced such that the remeshing may accommodate the need to resolve the sharp pressure gradient as well as the strain localization. The predicted responses are found to be not influenced by the mesh size due to the micromorphic regularization, while the adaptive meshing enables us to capture the width of deformation bands without the necessity of employing fine mesh everywhere in the domain.
\end{abstract}

(c) 2019 Elsevier B.V. All rights reserved.

Keywords: Adaptive meshing; Configurational force; Regularization; Poromechanics; Lie-algebra interpolation

\section{Introduction}

The path-dependent responses of geological materials, such as clay, sedimentary rock, limestone and crystalline rock are inherently anisotropic and size-dependent. These material characteristics can be captured, for example, via nonlocal models by incorporating the internal microstructure (e.g., [1-5]) or gradient plasticity models (e.g., [6-11]). Due to the introduction of a material length scale, the resultant computational models are able to circumvent the spurious mesh bias even in the post-bifurcation regimes where strain localization occurs. Nevertheless, capturing the solid skeleton responses during strain localization with a proper regularization mechanism is a necessary but

\footnotetext{
* Corresponding author.

E-mail address: wsun@columbia.edu (W. Sun).
} 
not sufficient condition to model the hydro-mechanical responses of the geological materials. During the formation of these deformation bands, pore fluid into the void space may repel from or flow into the deformation band and therefore leads to sharp pore pressure gradient. Reproducing these rapid changes of pore pressure and deformation is not only important for predicting the macroscopic mechanical responses of the geological system, but is equally important for replicating the hydro-mechanical responses both inside and outside the failure zone.

While there are theoretical frameworks that capture the size effect of metals with isochoric plastic flow (e.g., [7,11-16]), the applications of these frameworks often present additional challenges on the computational resources due to high demand of degree of freedom required to resolve the gradients and fluxes in a discretized spatial domain. Presumably, this can be solved by using a very fine spatial discretization related to the length scale of the material models. However, such a brutal-force approach is not always desirable in situations where (1) computational resources are limited and (2) the location of the domain of interest is not known a priori.

In this work, we introduce a unified mesh adaptation framework designed to address these issues for fluidinfiltrating porous media. Firstly, we introduce the micromorphic regularization for the anisotropic Cam-Clay model without significantly increasing the complexity of the formulation and implementation. To achieve these goals, we adopt the micromorphic energy functional in [10,17-21] to introduce size effect for the anisotropic critical state plasticity in [22] such that the size-dependent transverse isotropic responses are enforced elegantly with a projection operator whereas the nonlocality is enforced in an operator-split algorithm to minimize the implementation effect.

By employing this regularized model as a test bed, we exploit the nonlocality of the internal variables to introduce h-adaption with efficiency. To overcome the demand of a high mesh resolution typically for gradient plasticity model, we introduce a mesh adaptation strategy that does not require the energy estimate as criterion. Instead, we employ the configurational force theory to establish an energetic criterion for h-mesh adaption, in which the mesh-refinement criteria are obtained by a simple post-processing procedure. Here we derive a new configurational force for dissipative fluid-infiltrating porous materials that exhibits gradient-dependent plastic flow (cf., [23]). This configurational force takes account of the coupling energy terms associated with the total stress concept in the undrained limit as well as the energy flux due to the pressure gradient terms. The new concepts are compared to the strain energy term considering only the solid part, which is consistent with the effective stress in the drained limit condition. Following the remeshing, we also develop a technique to establish new equilibrium by mapping tensorial internal variables via Lie algebra to preserve the mechanical properties for path-dependent materials.

The organization of the rest of the paper is as follows. We first introduce the micromorphic anisotropic Cam-Clay model that introduces the size-dependent effect on the critical state and the hardening and describe the use of operator-split to simplify implementation of the constitutive law (Section 2). To meet the demand of resolution to resolve the gradient of the state variables, a refinement strategy that combines the advantages of the configurational force (as a re-meshing criterion) and the Lie algebra transfer (as projector of history-dependent variables) to establish equilibrium upon remeshing is presented (Section 3). To make the paper self-consistent, we then provide a brief review on the balance principle and the spatial and temporal discretizations of the poromechanics problems (Section 4). Finally, numerical examples are provided to demonstrate the performance of the proposed model in terms of consistency under the various mechanical and hydraulic loading conditions (Section 5). Finally, the major contribution and findings are summarized (Section 6).

As for notations and symbols, bold-faced letters denote tensors; the symbol ' ' denotes a single contraction of adjacent indices of two tensors (e.g., $\boldsymbol{a} \cdot \boldsymbol{b}=a_{i} b_{i}$ or $\boldsymbol{c} \cdot \boldsymbol{d}=c_{i j} d_{j k}$ ); the symbol ' $:$ ' denotes a double contraction of adjacent indices of tensor of rank two or higher (e.g., $\boldsymbol{C}: \boldsymbol{\epsilon}^{e}=C_{i j k l} \epsilon_{k l}^{e}$ ); the symbol ' $\otimes$ ' denotes a juxtaposition of two vectors (e.g., $\boldsymbol{a} \otimes \boldsymbol{b}=a_{i} b_{j}$ ) or two symmetric second order tensors (e.g., $(\boldsymbol{\alpha} \otimes \boldsymbol{\beta})_{i j k l}=\alpha_{i j} \beta_{k l}$ ). Moreover, $(\boldsymbol{\alpha} \oplus \boldsymbol{\beta})_{i j k l}=\alpha_{j l} \beta_{i k}$ and $(\boldsymbol{\alpha} \ominus \boldsymbol{\beta})_{i j k l}=\alpha_{i l} \beta_{j k}$. We also define identity tensors $(\boldsymbol{I})_{i j}=\delta_{i j},\left(\boldsymbol{I}^{4}\right)_{i j k l}=\delta_{i k} \delta_{j l}$, and $\left(\boldsymbol{I}_{\text {sym }}^{4}\right)_{i j k l}=\frac{1}{2}\left(\delta_{i k} \delta_{j l}+\delta_{i l} \delta_{k j}\right)$, where $\delta_{i j}$ is the Kronecker delta.

\section{Micromorphic regularization of the critical state plasticity}

We present the micromorphic regularization for critical state plasticity models derived to capture the plastic dilatancy and pore collapse in geomaterials under different confining pressures. The micromorphic regularization is following the strategy previously used in [19] where an additional global field variable $(\alpha)$ is constrained by an additional material force balance equation. This material force balance equation penalizes the discrepancy between the global field variable $(\alpha)$ and the local internal variable $(\bar{\alpha})$, while simultaneously regularizing the global field variable via introducing of a Laplacian term in the material force balance equation. The resultant constitutive law 
is then updated in a global-local operator-split setting such that the local internal variable is updated in the local constitutive laws at the material point for a given field variable $\alpha$, and the global internal variable is updated incrementally by solving the material force balance equation.

The key point in the local aspect is that the two variables are linked by a penalty term, in which the coupling is achieved by updating the internal variable $(\bar{\alpha})$ through the Newton's iteration. While the previous works mostly apply this method to von Mises-type elasto-plasticity by selecting the local equivalent plastic strain as $\bar{\alpha}$ (e.g., [18-21,24]), we present how this approach is applied to Cam-Clay type plasticity models. In the global aspect, the global and local variables are resolved together via the modified Helmholtz equation which introduces size effect associated with a plastic length scale.

\subsection{Nonlocal anisotropic modified Cam-Clay model}

We introduce a gradient regularization into an anisotropic version of the modified Cam-Clay model with a design that minimizes implementation effort. The anisotropic Cam-Clay model recently published in [22] is used as the basis model. The material is assumed to remain in the isothermal condition and hence heat transfer and thermal effect on hardening/softening is neglected. For simplicity, infinitesimal deformation is assumed such that the deformation is characterized by the infinitesimal strain $(\boldsymbol{\epsilon})$, the symmetric part of the gradient of the displacement field $(\boldsymbol{u})$, that is,

$$
\boldsymbol{\epsilon}=\nabla^{\mathrm{sym}} \boldsymbol{u}=\frac{1}{2}\left(\nabla \boldsymbol{u}+(\nabla \boldsymbol{u})^{\mathrm{T}}\right) .
$$

The additive decomposition of the infinitesimal strain leads to,

$$
\epsilon=\epsilon^{\mathrm{e}}+\epsilon^{\mathrm{p}},
$$

where $\epsilon^{\mathrm{e}}$ and $\epsilon^{\mathrm{p}}$ are the elastic and plastic splits of the strain, respectively. Assuming that the pore space remains fully saturated with one type of fluid, the effective stress-strain relationship can be written in general incremental form as,

$$
\Delta \sigma^{\prime}=\mathbb{C}: \Delta \epsilon,
$$

where $\mathbb{C}$ is a fourth-order tensor of tangential moduli.

For completeness, we first provide a brief review of the anisotropic modified Cam-Clay theory introduced in [22]. Based on the isotropic Modified Cam-Clay (MCC) theory [25], the anisotropic Cam-Clay model utilizes a linear mapping that maps anisotropic stress state into a fictitious isotropic stress state. This treatment then enables one to generate weighted invariants that take account of the orientation of the isotropic plane. Using these weighted invariants as replacement of the actual stress invariants, one can then express the nonlinear system of equations for the transversely isotropic plasticity model as a function of these weighted invariants instead of introducing principal directions or the components of stress tensors. Consequently, this treatment enables one to incorporate anisotropy in the existing return mapping algorithm intended for isotropic elasto-plasticity models (which are often written in the invariant form), without excessive implementation effort [26].

Considering the effective Cauchy stress $\left(\sigma^{\prime}\right)$, the two-invariant yield surface for the anisotropic Cam-Clay plasticity can be expressed as,

$$
f_{y}=\frac{q^{* 2}}{M^{2}}+p^{\prime *}\left(p^{\prime *}-p_{c}^{\prime}\right)=0,
$$

where $p_{c}^{\prime}<0$ is a plastic internal variable determining the size of yield surface, that is known as the pre-consolidation pressure. The mapped mean stress $p^{\prime *}$ and deviatoric stress $q^{*}$ are defined by,

$$
p^{*}=\boldsymbol{a}^{*}: \boldsymbol{\sigma}^{\prime}, \quad q^{* 2}=\frac{1}{2} \boldsymbol{\sigma}^{\prime}: \boldsymbol{A}^{*}: \boldsymbol{\sigma}^{\prime}
$$

where $\boldsymbol{a}^{*}=\boldsymbol{I} / 3$ and $\boldsymbol{A}^{*}=\boldsymbol{P}^{\mathrm{p}}:(3 \boldsymbol{D}): \boldsymbol{P}^{\mathrm{p}}$, for $\boldsymbol{D}=\boldsymbol{I}^{4}-\boldsymbol{I} \otimes \boldsymbol{I} / 3$. Here the mapping tensor $\boldsymbol{P}^{\mathrm{p}}$ is

$$
\begin{aligned}
P_{i j k l}^{\mathrm{p}} & =\frac{c_{1}^{\mathrm{p}}}{2}\left(\delta_{i k} \delta_{j l}+\delta_{i l} \delta_{j k}\right)+\frac{c_{2}^{\mathrm{p}}}{2}\left(\phi_{i k} \phi_{j l}+\phi_{i l} \phi_{j k}\right) \\
& +\frac{c_{3}^{\mathrm{p}}}{4}\left(\delta_{i k} \phi_{j l}+\delta_{i l} \phi_{j k}+\phi_{i k} \delta_{j l}+\phi_{i l} \delta_{j k}\right),
\end{aligned}
$$


where $c_{1}^{\mathrm{p}}$ through $c_{3}^{\mathrm{p}}$ are fourth-order tensor coefficients related to input material parameters $\alpha^{\mathrm{p}}, \beta^{\mathrm{p}}$, and $\gamma^{\mathrm{p}}$. The relations are $c_{1}^{\mathrm{p}}=\beta^{\mathrm{p}}, c_{2}^{\mathrm{p}}=\alpha^{\mathrm{p}}+\beta^{\mathrm{p}}-2 \gamma^{\mathrm{p}}$, and $c_{3}^{\mathrm{p}}=2\left(\gamma^{\mathrm{p}}-\beta^{\mathrm{p}}\right)$. We note that the isotropy of the MCC plastic response is recovered under $\boldsymbol{P}^{\mathrm{p}}=\boldsymbol{I}^{4}$, hence $\alpha^{\mathrm{p}}=\beta^{\mathrm{p}}=\gamma^{\mathrm{p}}=1$. Interested readers can refer to Semnani et al. [22] for details.

The hardening law for the Cam-Clay type models accommodates the bilogarithmic relationship between the specific volume and the preconsolidation pressure under the condition of $0<c_{r}<c_{c}$ (e.g., [22,27-31]), that is,

$$
\dot{p}_{c}^{\prime}=-\frac{\dot{\epsilon}_{v}^{\mathrm{p}}}{\lambda_{\mathrm{p}}} p_{c}^{\prime},
$$

where $\epsilon_{v}^{\mathrm{p}}$ indicates the plastic volumetric strain; $\lambda_{\mathrm{p}}=c_{c}-c_{r}$ with $c_{c}$ the total compressibility index and $c_{r}$ the elastic compressibility index. Therefore, the internal variable $p_{c}^{\prime}$ is updated according to the corresponding hardening law. In other words, the constitutive models of the Cam-Clay type with the hardening law of Eq. (7) acquire the incremental plastic strain by (1) obtaining the volumetric component of plastic strain via the hardening law for $p_{c}^{\prime}$ then (2) using the plastic dilatancy to obtain the deviatoric invariant of the plastic strain increment along the flow direction such that the stress can be updated incrementally [31]. In the gradient-enhanced anisotropic Cam-Clay model, we modify the increment form of the hardening rule by introducing a penalty term $\left(\varepsilon_{p}\right)$ such that,

$$
f_{r}=p_{c}^{\prime}-p_{c, \text { old }}^{\prime} \exp \left(\frac{\epsilon_{v}^{\mathrm{e}}-\epsilon_{v}^{\mathrm{e}}}{\lambda_{\mathrm{p}}}\right)+\varepsilon_{p}\left(p_{c}^{\prime}-\alpha\right)=0 .
$$

Here we consider the hardening variable $p_{c}^{\prime}$ as a local internal variable $\bar{\alpha}\left(p_{c}^{\prime} \equiv \bar{\alpha}\right)$. Thus, $\alpha$ denotes a global micromorphic variable associated with nonlocal $p_{c}^{\prime}$, which is used in the micromorphic balance equation (10) for nonlocal setting. By appending this modified hardening equation (8) to the local residual vector (9), the coupling between the local and global variables is achieved through a return mapping algorithm. Note again that the local internal variable $\bar{\alpha}$ is equal to $p_{c}^{\prime}$, which is linked to the global micromorphic variable $\alpha$ by the penalty parameter $\varepsilon_{p}$. The local nonlinear systems, therefore, are assembled by the local residual vector $(\boldsymbol{r})$ and the unknown vector $(\boldsymbol{x})$,

$$
\boldsymbol{r}=\left\{\begin{array}{c}
\boldsymbol{\epsilon}^{\mathrm{e}}-\boldsymbol{\epsilon}^{\mathrm{e} \text { trial }}+\Delta \lambda \partial_{\sigma} f \\
f_{y} \\
f_{r}
\end{array}\right\}_{8 x 1} ; \quad \boldsymbol{x}=\left\{\begin{array}{c}
\boldsymbol{\epsilon}^{\mathrm{e}} \\
\Delta \lambda \\
p_{c}^{\prime}
\end{array}\right\}_{8 x 1} ; \quad \boldsymbol{a}=\boldsymbol{r}^{\prime}(\boldsymbol{x}),
$$

which is solved by a local Newton's iteration approach. $\epsilon^{\mathrm{e}}$ is an ordered list of strain components. More details about the process of the return mapping algorithm can be found from the previous work [22].

\subsection{Introducing regularization via a local-global operator split}

We introduce the micromorphic regularization for a constitutive law designed to capture the local stress-strain response via a penalty method, in which one introduces two additional energy functionals for the variational constitutive updates: the first as the prime variable in the Helmholtz equation whose gradient term is constrained by the Laplacian operator; and, the other generates penalty energy that controls the discrepancy between the internal variable of the local constitutive laws to the prime variable of the Helmholtz equation. This method has been previously employed in, for instance, Forest [21], Aldakheel and Miehe [24], Miehe et al. [18].

Here we denote the micromorphic variable as $\alpha$. Introducing local internal variable $\bar{\alpha}$ into the modified Helmholtz equation constrains the difference between the micromorphic variable and the local internal variable (cf., [21,32-35]), that is,

$$
\alpha-l_{p}^{2} \Delta \alpha=\bar{\alpha},
$$

where the regularization is established through the characteristic (plastic) length scale $l_{p}$. Note that $l_{p}$ not only constitutes as the characteristic length of the materials, but also prevents mesh sensitivity upon strain localization [11,36-38]. In this work, we introduce gradient dependence to the local preconsolidation pressure $p_{c}^{\prime}$ such that $p_{c}^{\prime}=\bar{\alpha}$. As pointed out by Forest [21], one key benefit of this indirect introduction of gradient dependence (comparing with the alternative which directly introduces gradient dependence into the hardening law Eq. (7) i.e. $\left.\dot{p}_{c}=\operatorname{tr}\left(\dot{\epsilon}^{p}\right) /\left(c_{c}-c_{r}\right) p_{c}\right)$ is the ease of implementation. As the global micromorphic variable $\alpha$ may exist 
everywhere in the domain, there is no need to explicitly locate the plastic zone for the gradient-dependent plastic flow, unlike the direct approach [17,19-21,24,32,35]. Here, we assume that the associated von Neumann boundary condition for the micromorphic variable always remain trivial, such that,

$$
\nabla \alpha \cdot \boldsymbol{n}=0 \text { on } \partial \mathcal{B}
$$

where only the global fields are restricted explicitly by this boundary condition.

We adopt the operator-split approach to solve the numerical solution incrementally. The incremental weak forms of the momentum and mass balance equations are solved monolithically to obtain the updated displacement and pore pressure fields. Following this step, we then obtain the incremental update of the micromorphic variable $\alpha$ for the micromorphic regularization This approach simplifies the derivation of the local Jacobian for Newton's iteration and the global consistent linearization procedure [10]. However, the incremental time step must be sufficiently small to maintain consistency among the governing equations. The spatial discretization and the solution strategy for resolving the problem via finite element analysis are covered in a later section (Section 4).

\section{Property-preserving adaptive re-meshing}

In this section, we introduce a mesh adaption procedure designed to enhance computational efficiency of the finite element simulations by leveraging the nonlocality of the gradient-enhanced plasticity model in the preceding section. We note that this procedure can be associated with other material models (local or nonlocal) as well. While increasing the numbers of degrees of freedom uniformly is an obvious remedy to improve the quality of finite element solutions (e.g., [39]), this approach demands significant computational resource and is inefficient to capture the localization of deformation upon material bifurcation. Furthermore, modeling strain localization with conventional finite element method often leads to pathological mesh dependence by strain softening (e.g., [40]). Therefore, an adaptive mesh refinement algorithm that can identify the critical regions (i.e. shear, compaction, and dilation bands in sand, clay, and rocks) is often found to be more efficient for the strain localization problems (e.g., [41-43]). Here our focus lies on how to properly perform the mesh refinement on problems involving historydependent materials characterized by gradient-dependent plastic flow. The key departure of this work is that we also incorporate the influence of the nonlocal energy functionals that generate the gradient-dependent plastic flow in our calculation of configurational force.

In the following sub-sections, we provide the three major ingredients necessary for continuing the simulations upon remeshing, i.e.: (1) the mesh refinement criteria (Section 3.1); (2) the transfer of internal variables in Lie group, including scalars and tensors (Section 3.2); and, (3) the re-establishment of equilibrium after the projection of internal variables (Section 3.3).

\subsection{Configurational force based refinement criteria}

The first key necessary ingredient for an adaptive meshing scheme is a suitable criteria for mesh refinement. This problem is closely related to how to identify the critical regions or singularities, such as strain localization and fracture, where the accuracy of the finite element approximation is diminished. Traditionally, significant effort has been devoted to deriving the error estimates for finite element solutions, where the major focus was on posteriori error estimators, for example, the pioneering work by Babuvška and Rheinboldt [44] and a series of works after [45]. Interested readers can refer to a vast amount of literature on posteriori error estimates (e.g., [42,46-50]). Based upon the energy minimization concept, Mosler and Ortiz [51], Mosler and Ortiz [52] incorporate the variational principle into the h-adaptive strategy which naturally drives mesh adaptation. This variational adaptivity is closely linked to configurational force balance, that has been used as an indicator for adaptive meshing scheme (e.g., [53-55]). In this study, we take advantages of the configurational force to define a threshold value for adaptive mesh refinement.

The configurational force, or equivalently termed material force, is associated with "the material's configurational changes" and influences the evolution or phase transition of material structures [56]. This concept, therefore, has been used to various applications in computational mechanics, such as defects, fracture, inclusions, and mesh discretization (e.g., [57,58]). For adaptive mesh refinement, we utilize discrete configurational forces introduced by finite element discretization [53,57]. The motivation of using the discrete configuration forces for adaptive meshing is two-fold. First, the discrete configurational forces can be used to identify the critical regions that require mesh refinement. We note that the derivation of discrete configurational forces is consistent with the finite element 
approximation of governing equations for the system [57]. In other words, the configurational forces change in the discretization, and theoretically, become zero for a homogeneous body without body forces. Therefore, nonvanishing configuration forces are considered to be the indicator for mesh refinement (e.g., [50]). Second, the configurational force balance is obtained as the post processing step. Since the energy-momentum tensor that comprises the configurational forces is derived from the finite element solutions, the mesh-refinement criteria can be calculated with a simple procedure (e.g., [55]).

In this study, we adopt the concept of configurational forces and extend it to incorporate poromechanics theory. The major interest lies in how the interaction between solid and fluid phases affect the configuration forces as mesh refinement criteria (e.g., [23]). Among the various derivations for configurational force balance, we used the relation of invariance properties, specifically, the translational invariance followed by Buggisch et al. [59], Mueller et al. [55]. The general structure of balance laws associated with the translational invariance of the control volume is described in [55] and hence not repeated in work. For simplicity, we limit our analysis in the small-strain assumption and quasi-static loading conditions.

\subsubsection{Configurational poromechanics for fully saturated porous media}

The configurational force has been used as a mesh refinement criterion (e.g., [58,60,61]) for more than a decade. Recently, Papastavrou and Steinmann [23] provide the derivations and assumptions that lead to different types of configurational forces for two-phase fluid-infiltrating porous media. In this work, our contribution is to provide a new derivation for the configurational force of poromechanics systems and extend it to micromorphic porous media where contributions of configurational force from the nonlocal functional that introduces gradient dependence to the local internal variables are taken into account. We introduce this new derivation of configurational force for the fully saturated porous media by deriving the configurational force based on the translational invariance of a control volume [55].

As a starting point, we consider the stored energy of a control volume of an apparent solid skeleton (cf., [62-64]). First introduced in [62], this apparent solid skeleton differs from the solid skeleton or the complete two-phase mixture defined in the classical theory of porous media in the sense that the apparent solid skeleton considers the energy exchange between the solid constituent and surface energy of the solid-fluid interface, but only considers the control volume of the solid skeleton. As such, it is convenient for considering the energy flux of the apparent solid skeleton along the trajectory of the solid skeleton. The energy of the apparent solid skeleton therefore reads [62-64],

$$
W=W_{\text {solid }}+W_{\text {fluid }}
$$

where $W_{\text {solid }}$ denotes the free energy stored in solid skeleton, and $W_{\text {fluid }}$ is contribution of free energy from the bulk fluid to the solid-fluid interface. $W_{\text {solid }}$ is further decomposed into its elastic and plastic components $W_{\text {solid }}=W^{\mathrm{e}}+W^{\mathrm{p}}$. In this study, the linear elasticity model is employed such that the elastic strain energy $\left(W^{\mathrm{e}}\right)$ is expressed as,

$$
W^{\mathrm{e}}=\frac{1}{2} \epsilon^{\mathrm{e}}: \mathbb{C}^{\mathrm{e}}: \epsilon^{\mathrm{e}}
$$

where $\mathbb{C}^{\mathrm{e}}$ is a fourth-order tensor that represents elastic response of a transversely isotropic material. The detailed components of $\mathbb{C}^{\mathrm{e}}$ are referred to Semnani et al. [22], Walpole [65], Crook et al. [66]. Due to the coupling between the deviatoric and volumetric plastic strain in the Cam-Clay plasticity theory, the rate of change of the local plastic work $\left(W^{\mathrm{p}}\right)$ can be expressed as (cf., [27] Eq. (6.20)),

$$
\Delta W^{\mathrm{p}}=\Delta \lambda p^{\prime *} p_{c}^{\prime} .
$$

In practice, this plastic work is computed incrementally via the backward Euler method. Notice that (14) is specific for Cam-Clay models in which the plastic dilatancy introduces a coupling between the plastic work done due to the plastic deviatoric and volumetric strain and hence greatly simplify the expression of the plastic work. More general discussion on the specific form of plastic work for other types of materials can be found in, for instance, Ortiz and Stainier [67], Weinberg et al. [68], Mosler and Bruhns [69], Miehe et al. [17] but is out of the scope of this study.

Next, the fluid contribution of the free energy $W_{\text {fluid }}$ takes the following form (cf., $\left.[23,64,70]\right)$,

$$
W_{\text {fluid }}=-B p \epsilon_{v}-\frac{1}{2 M} p^{2},
$$


where $p$ is the pore pressure field; $\epsilon_{v}$ denotes the volumetric strain; $B$ is the Biot's coefficient; $M$ is the Biot's modulus. Based on the additive decomposition of the strain measure (2), $\epsilon_{v}$ can be rewritten as,

$$
\begin{aligned}
\epsilon_{v} & =\boldsymbol{I}: \boldsymbol{\epsilon}=\boldsymbol{I}:\left(\boldsymbol{\epsilon}^{\mathrm{e}}+\boldsymbol{\epsilon}^{\mathrm{p}}\right) \\
& =\epsilon_{v}^{\mathrm{e}}+\epsilon_{v}^{\mathrm{p}} .
\end{aligned}
$$

In other words, the volumetric strain can be additively decomposed into elastic and plastic components (cf., [70]). Therefore, the stored energy function $\left(W=W_{\text {solid }}+W_{\text {fluid }}\right)$ is then used to derive the configurational force of poromechanics system, following the treatment in [55].

Now consider a reference domain $\Omega$ and its boundary $\partial \Omega$. In addition to contributions of the free energy described above (12), the coupling between the local and micromorphic internal variables and gradient dependence of the plastic flow introduced via the micromorphic internal variable also constitutes energy. As a result, the resultant regularized energy-dissipation functional for the apparent solid skeleton reads (cf., $[16,64,71]$ ),

$$
\begin{aligned}
\mathscr{F} & =\int_{\Omega} \underbrace{\left(W_{\text {solid }}+W_{\text {fluid }}\right)}_{\phi} d V-\int_{\partial_{t} \Omega} \overline{\boldsymbol{t}} \cdot \boldsymbol{u} d A-\int_{\Omega} \boldsymbol{r} \cdot \boldsymbol{u} d V \\
& -\int_{0}^{t}\left[\int_{\partial_{q} \Omega} \bar{q} p d A+\int_{\Omega} \bar{s} p d V\right] d \tau+\underbrace{\int_{0}^{t}\left[\int_{\Omega} \frac{1}{2 \mu} \boldsymbol{k}:(\nabla p \otimes \nabla p) d V\right] d \tau}_{\mathcal{D}_{f}} \\
& +\int_{\Omega}^{\left[\frac{1}{2\left|p_{c_{0}}^{\prime}\right|} l_{m}^{2}\|\nabla \alpha\|^{2}+\frac{\varepsilon_{p}}{2\left|p_{c_{0}}^{\prime}\right|}(\bar{\alpha}-\alpha)^{2}\right]} d V .
\end{aligned}
$$

Here $\phi$ denotes the stored energy of the fluid-infiltrating porous media; $\mathcal{D}_{f}$ is the dissipation associated with the Darcian flow of the pore fluid; $\overline{\boldsymbol{t}}=\boldsymbol{\sigma}^{\prime} \cdot \boldsymbol{n}$ is the specified traction; $\bar{q}=-\boldsymbol{n} \cdot \tilde{\boldsymbol{v}}$ is the specified flux; $\boldsymbol{r}$ and $\bar{s}$ are source terms; $\boldsymbol{k}$ is intrinsic permeability tensor; $\mu$ is viscosity; $\phi_{m}$ is defined as the micromorphic energy function; $l_{m}$ is a micromorphic length scale related to the gradient of the global micromorphic variable $\alpha ; \epsilon_{p}$ is the penalty parameter; $p_{c_{0}}^{\prime}$ is the initial preconsolidation stress, which is introduced to maintain consistent units $(|\cdot|$ is the absolute value of the given $p_{c_{0}}^{\prime}$ ). We note that the characteristic (plastic) length scale $l_{p}$ in (10) can be defined as $l_{p}:=\sqrt{l_{m}^{2} / \varepsilon_{p}}$, where $\varepsilon_{p}$ is non-dimensional. While this functional (17) gives rise to derive the governing equations (35) to (37) for fluid-saturated materials associated with the micromorphic regularization, the micromorphic energy function $\phi_{m}$ is not considered in deriving configurational force below to exclude any nonlocal contribution. The influence of $\phi_{m}$ on configurational force and its meaning, however, will be discussed later (Section 5.1).

The energy balance equation for saturated porous media (excluding the micromorphic contribution $\left(\phi_{m}\right)$ ), reads,

$$
\begin{aligned}
& \frac{d}{d t} \int_{\Omega}\left(W_{\text {solid }}+W_{\text {fluid }}\right) d V \\
& \quad=\int_{\partial \Omega}\left(\boldsymbol{\sigma}^{\prime} \cdot \boldsymbol{n}\right) \cdot \boldsymbol{v} d A+\int_{\Omega} \boldsymbol{r} \cdot \boldsymbol{v} d V-\int_{\partial \Omega} p \tilde{\boldsymbol{v}} \cdot \boldsymbol{n} d A+\int_{\Omega} \bar{s} p d V-\int_{\Omega}\left[\frac{1}{2 \mu} \boldsymbol{k}:(\nabla p \otimes \nabla p)\right] d V .
\end{aligned}
$$

without the source terms $\bar{s}$ and $\boldsymbol{r}$, the expression of Eq. (18) can be simplified by introducing the following terms,

$$
\begin{aligned}
& \phi=W_{\text {solid }}+W_{\text {fluid }}, \quad \boldsymbol{\Phi}_{\text {solid }}=\boldsymbol{\sigma}^{\prime} \cdot \boldsymbol{v}, \\
& \boldsymbol{\Phi}_{\text {fluid }}=-p \tilde{\boldsymbol{v}}, \quad R=\frac{1}{2 \mu} \boldsymbol{k}:(\nabla p \otimes \nabla p) .
\end{aligned}
$$

Integrating $\boldsymbol{H}=(\dot{\phi}-R) \boldsymbol{I}-(\nabla \boldsymbol{\Phi})^{\mathrm{T}}$ with respect to time yields,

$$
\mathbb{I}=\int_{0}^{t} \boldsymbol{H} d \tau
$$

Or expressed by index notation for Cartesian coordinates,

$$
\mathbb{I}_{i j}=\phi \delta_{i j}-\int_{0}^{t}\left(\sigma_{j k}^{\prime} v_{k}\right)_{, i} d \tau+\int_{0}^{t}\left(p \tilde{v}_{j}\right)_{, i} d \tau-\left(\int_{0}^{t} \frac{1}{2 \mu} k_{l m} p_{, l} p_{, m} d \tau\right) \delta_{i j}
$$


Here the second integrand can be reformulated as follows,

$$
\begin{aligned}
\left(\sigma_{j k}^{\prime} v_{k}\right)_{i} & =\sigma_{j k, i}^{\prime} v_{k}+\sigma_{j k}^{\prime} \dot{u}_{k, i} \\
& =\frac{\dot{u_{k, i} \sigma_{j k}^{\prime}}}{u_{k, i} \dot{\sigma}_{j k}^{\prime}+\sigma_{j k, i}^{\prime} v_{k} .}
\end{aligned}
$$

Substituting (22) into (21) yields,

$$
\mathbb{I}_{i j}=\underbrace{\phi \delta_{i j}-u_{k, i} \sigma_{j k}^{\prime}}_{\Sigma_{i j}}+\int_{0}^{t}\left(u_{k, i} \dot{\sigma}_{j k}-\dot{u}_{k, i} \sigma_{j k}^{\prime}\right) d \tau+\int_{0}^{t}\left(p \tilde{v}_{j}\right)_{, i} d \tau-\left(\int_{0}^{t} \frac{1}{2 \mu} k_{l m} p_{, l} p_{, m} d \tau\right) \delta_{i j} .
$$

Here the notation $\boldsymbol{\Sigma}=\phi \boldsymbol{I}-(\nabla \boldsymbol{u})^{\mathrm{T}} \cdot \boldsymbol{\sigma}^{\prime}$ is used, which is often called as the energy-momentum tensor, or NewtonEshelby stress tensor. Before we interpret the derivation in terms of poromechanics, we note again that the analysis is limited under the static condition. This can be also considered as the special case of dynamic condition that is a plane stationary wave or standing wave condition. In static case, the second term in (23) becomes zero (cf., [59]): $u_{k, i} \dot{\sigma}^{\prime}{ }_{j k}-\dot{u}_{k, i} \sigma_{j k}^{\prime}=0$. Therefore, (23) can be rewritten as,

$$
\mathbb{I}_{i j}=\underbrace{\phi \delta_{i j}-u_{k, i} \sigma_{j k}^{\prime}}_{\Sigma_{i j}}+\int_{0}^{t}\left(p \tilde{v}_{j}\right)_{, i} d \tau-\left(\int_{0}^{t} \frac{1}{2 \mu} k_{l m} p_{, l} p_{, m} d \tau\right) \delta_{i j} .
$$

Now that we have derived the expression of $\mathbb{I}$ associated with poromechanics theory, its meaning may be interpreted by the assumptions we make, which will be discussed in the next section.

We recall the micromorphic energy $\phi_{m}$ in (17). Followed by the derivation of the energy-momentum tensor $\mathbb{I}, \phi_{m}$ can be considered as an additional term of $\phi$ in (24) that constitutes the energy-momentum tensor $\boldsymbol{\Sigma}$. As the micromorphic energy indicates the change of local $(\bar{\alpha})$ and global $(\alpha)$ variables associated with the preconsolidation pressure $p_{c}^{\prime}$, we infer that this term $\phi_{m}$ is closely related to the plastic behavior. The effect of $\phi_{m}$ on the mesh-refinement criteria using the configurational force will be discussed in the numerical example sections (Section 5).

\subsubsection{Configurational force in idealized conditions}

For simplicity, we may first consider the fully drained state in which no pore pressure is concerned. Naturally, the last two terms in (24) are ignored, which results in only the energy-momentum tensor as,

$$
\mathbb{I}=\boldsymbol{\Sigma}=W_{\text {solid }} \boldsymbol{I}-(\nabla \boldsymbol{u})^{\mathrm{T}} \cdot \boldsymbol{\sigma}^{\prime} .
$$

As $W_{\text {solid }}$ denotes the effective stain energy by assuming the drained limit state, this equation (25) is consistent with the solid only condition. As discussed by Mueller et al. [55], the divergence of the energy-momentum tensor $\boldsymbol{\Sigma}$, or the material volume force, vanishes for a homogeneous body without physical volume forces, which is the central idea using the configurational force as refinement criteria in a finite element framework.

In terms of poromechanics theory, we then consider the undrained limit in which the pore-fluid remains trapped inside pores. By further excluding the specified flux $\bar{q}$, the energy-momentum tensor can be expressed as,

$$
\mathbb{I}=\boldsymbol{\Sigma}=\left(W_{\text {solid }}-B p \epsilon_{v}-\frac{1}{2 M} p^{2}\right) \boldsymbol{I}-(\nabla \boldsymbol{u})^{\mathrm{T}} \cdot \boldsymbol{\sigma}^{\prime} .
$$

By introducing incompressibility of solid and fluid constituents and assuming the Biot's coefficient as $B=1$, the expression (26) is simplified as,

$$
\mathbb{I}=\boldsymbol{\Sigma}=\left(W_{\text {solid }}-p \epsilon_{v}\right) \boldsymbol{I}-(\nabla \boldsymbol{u})^{\mathrm{T}} \cdot \boldsymbol{\sigma}^{\prime} .
$$

As an extension, we may further consider the diffusion of pore fluid by considering the dissipation term $D_{f}$ as,

$$
\mathbb{I}=(\underbrace{W_{\text {solid }}-p \epsilon_{v}}_{W_{\text {solid }}+W_{\text {fluid }}}-\underbrace{\int_{0}^{t}\left[\frac{1}{2 \mu} \boldsymbol{k}:(\nabla p \otimes \nabla p)\right] d \tau}_{\mathcal{D}_{f}}) \boldsymbol{I}-(\nabla \boldsymbol{u})^{\mathrm{T}} \cdot \boldsymbol{\sigma}^{\prime},
$$


which includes the time-dependent dissipative influence on the calculation of configurational force. Again, $W_{\text {solid }}$ denotes the strain energy term; $W_{\text {fluid }}$ is the simplified stored energy term in fluid; and $\mathcal{D}_{f}$ is the flow diffusion energy term.

Once we obtain the energy-momentum tensor $\mathbb{I}$, we may follow the derivation of the discrete material forces as,

$$
\boldsymbol{G}^{I}=\int_{\mathcal{B}_{e}} \mathbb{I} \cdot \boldsymbol{D} d V
$$

which is consistent extension from [55]. Note that $\boldsymbol{D}=\nabla \lambda_{a}$ indicates the gradient of the test function or the shape function. The discrete material forces are obtained from the existing solutions at the equilibrium state. In other words, the force calculation can be considered as a post-processing procedure. For adaptive mesh refinement, we can set the criteria by adopting the magnitude of $G^{I}=\left\|G^{I}\right\|$, where $\|\cdot\|$ indicates $L^{2}$ norm of the given vector.

\subsection{Transfer operation of internal variables via Lie algebra}

Generally speaking, re-meshing for simulations dealing with path-dependent materials is more complicated than the path-independent counterpart. The reason is that any mesh adaptation would require a reconstruction of equilibrium state after the mesh is refined. In other words, the information from the numerical simulations in the old mesh must be transferred into the new mesh before the new incremental update is sought. For physical qualities that are directly related to the nodal variables, this reconstruction simply requires a new interpolation using a new set of basis functions associated with the new mesh.

However, for most problems that deal with path-dependent responses, one cannot establish a new equilibrium state without considering the history of state variables. This can become an important issue for re-meshing as any modification of the mesh may lead to a new set of integration point at locations different from those in the old mesh. Without knowing explicitly the loading history of these new material points, the only viable alternative left is to extrapolate all the state variables that enables us to approximate the history-dependent behaviors. It is therefore not difficult to imagine that an inaccurate extrapolation of the state variables could both cause significant errors and make it impossible to establish the new equilibrium in the new mesh.

In this work, we employ a property-preserving Lie algebra mapping to extrapolate scalar and tensorial state variables. While there are previous works that employ similar strategy for the recovery of stress [72] and the internal variables in finite strain regime [73,74], our works actually find that the Lie algebra mapping can also be crucial in the small-deformation regime, especially when the residuals of the equilibrium equations are highly sensitive to perturbation of internal variables (e.g., strain softening). We compute the spectral decomposition of the tensor and obtain the logarithms of the rotation and stretch component separately. Note that eigenvectors obtained from the spectral decomposition constitute the column vectors of the rotation tensor $\boldsymbol{R}$. We then interpolate each scalar component and apply the exponential to recombine the tensor variable.

The logarithm of a rotation can be computed using the explicit formulas $[73,75]$. Firstly, the angle of rotation $(\theta)$ is obtained by,

$$
\theta:=\cos ^{-1}\left[\frac{1}{2}(\operatorname{tr} \boldsymbol{R}-1)\right], \quad \boldsymbol{R} \in S O(3), \quad \theta \in[0, \pi] .
$$

Depending on the $\theta$, the logarithm of the rotation is determined as follows,

$$
\log \boldsymbol{R}= \begin{cases}\boldsymbol{0} & \text { if } \theta=0, \\ \frac{\theta}{2 \sin \theta}\left(\boldsymbol{R}-\boldsymbol{R}^{\mathrm{T}}\right) & \text { if } \theta \in(0, \pi), \\ \pm \pi \check{\boldsymbol{v}} & \text { if } \theta=\pi,\end{cases}
$$

Here $\check{\boldsymbol{v}}$ is the skew-symmetric tensor such that $\check{\boldsymbol{v}} \cdot \boldsymbol{u} \equiv \boldsymbol{v} \times \boldsymbol{u} \forall \boldsymbol{u} \in \mathbb{R}^{3}, \boldsymbol{v}$ is the eigenvector corresponding to the eigenvalue of 1 of $\boldsymbol{R}$, and the sign is selected according to continuity from the field in the neighborhood. The sign of the exponential map can be selected likewise [73,75]. Let $\boldsymbol{W} \in \operatorname{so}(3)$, we define the angle of rotation as follows,

$$
\theta:=\left(\frac{\boldsymbol{W}: \boldsymbol{W}}{2}\right)^{\frac{1}{2}} \text {. }
$$




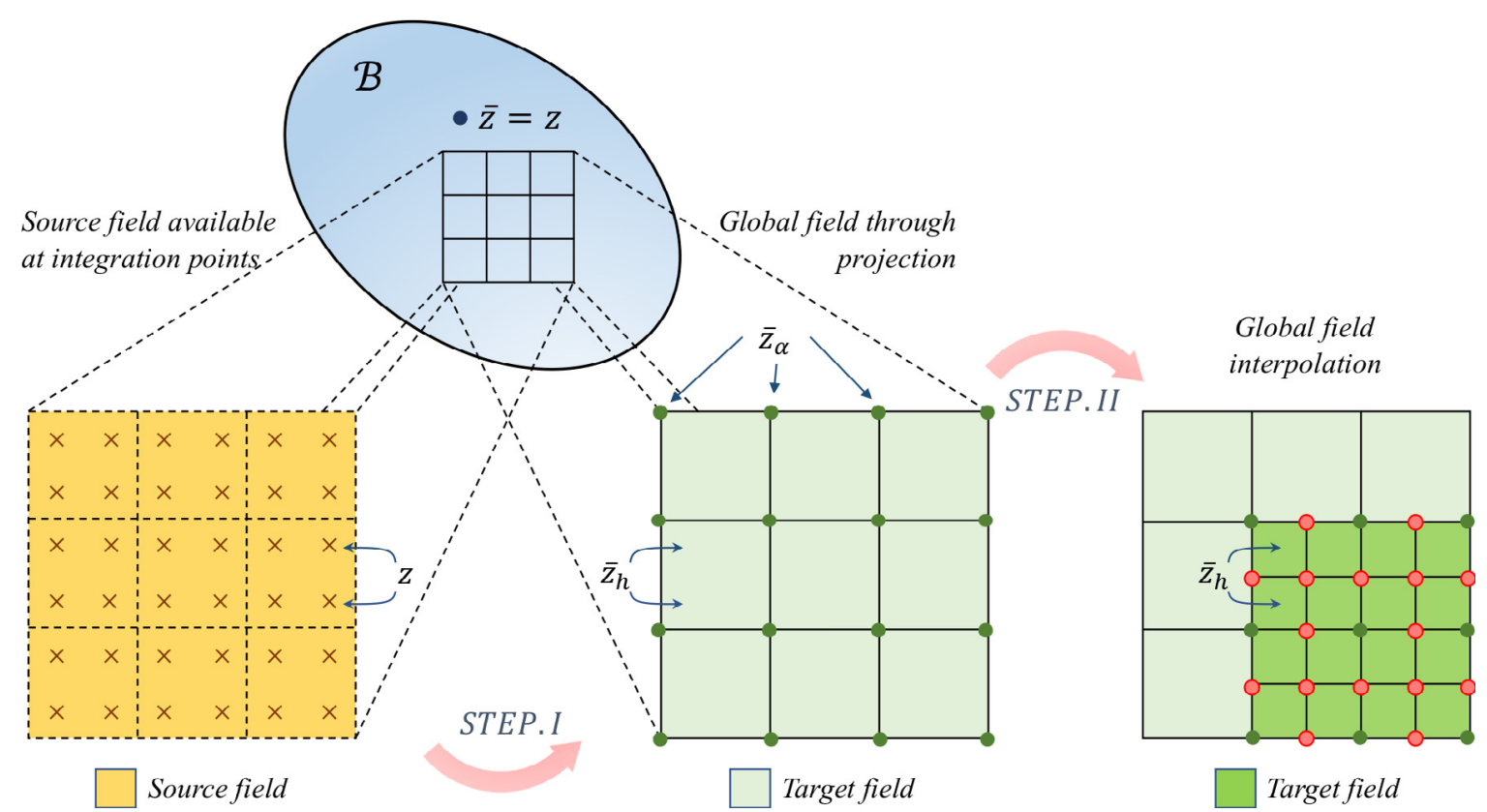

Fig. 1. Projection of integration-point values of the source field into nodal values of the discrete target field (STEP.I: projection of source fields to nodal values; STEP.2: interpolation of nodal values onto the new mesh).

Source: Modified from [73].

The exponential map for the skew-symmetric tensor $\boldsymbol{W}$ then is given by the following expression,

$$
\exp \boldsymbol{W}= \begin{cases}\boldsymbol{I}, & \text { if } \theta=0 \\ \boldsymbol{I}+\frac{\sin \theta}{\theta} \boldsymbol{W}+\frac{(1-\cos \theta)}{\theta^{2}} \boldsymbol{W}^{2}, & \text { if } \theta>0\end{cases}
$$

The projection of the components, the logarithms of the rotation and stretch components, are conducted using $L_{2}$ projection. As proved in [73], the projection of internal variables using $L_{2}$ is derived naturally based upon the variational approach. Furthermore, the global $L_{2}$ projection leads to smooth linear field, which is identical to the linear least squares regression of the nodal values of the original source field [73]. The recovery of target field $\bar{z}_{h}$ by nodal values $\bar{z}_{\alpha}$, the interpolation functions $\lambda_{\alpha}$, and the source field $z$ can be expressed by,

$$
\begin{aligned}
& \bar{z}_{h}(\boldsymbol{X}):=\lambda_{\alpha}(\boldsymbol{X}) \bar{z}_{\alpha} \\
& \bar{z}_{h}=\lambda_{\alpha}\left(\int_{\mathcal{B}} \lambda_{\alpha} \lambda_{\beta} \boldsymbol{I} d V\right)^{-1} \int_{\mathcal{B}} \lambda_{\beta} z d V .
\end{aligned}
$$

To compute the integrals, the interpolation functions $\lambda_{\alpha}$ and $\lambda_{\beta}$ are adopted. The proof that the projection is optimal is referred to Mota et al. [73]. Fig. 1 describes the concept of the projection procedure. As the source fields, or internal variables, are located at the integration, these values are projected on the nodal points by $L^{2}$ projection (34) before the mesh is refined. Using the nodal values in the original mesh, the new values at the refined mesh can be obtained as well, which leads to recover the source fields at the new integration points (e.g., $[41,51,76])$. One of the potential issues in the mesh-refinement is the treatment of hanging nodes. Here we leverage the open-source finite element package, Deal.II, to ensure that the degrees of freedom on hanging nodes conform to constraints such that the global solution is continuous. Interested readers are referred to Bangerth et al. $[77,78]$ and literature therein.

\subsection{Reestablishment of equilibrium after mesh refinement}

Once we recover the prime variables and internal variables onto the new mesh, the re-establishment of equilibrium completes the adaptive mesh refinement process. Because the mesh refinement is triggered by the post-processing 
procedure, the re-equilibrium can be sought from the solutions that are in the equilibrium state. Our focus is therefore to remap all the variables properly so that the converged solution on the new mesh is consistent with that of the original mesh. In other words, once the adaptivity criterion is met (Section 3.1) at the numerical step of $n$, the mesh refinement followed by the state updates on the new mesh is performed from a fixed set of initial conditions at the step $n$. The mesh adaption and global iteration stop when the refinement is not triggered for the converged solution.

As our focus lies in the mesh refinement techniques for history-dependent materials, the accuracy of recovering the state variables is critical to maintain the consistency. If the remapping procedure recovers the exact states in the refined mesh from the original mesh condition, for example, the iteration will be unnecessary to reestablish the equilibrium. In reality, however, the interpolation error always occurs due to the nature of admissible space of solutions in finite element approach [41]. Besides, the loss of ellipticity in governing equations leads to mesh dependent results under the strain localization problems. Because the regularization is introduced via the micromorphic approach, therefore, our strategy is to obtain the state variables of the new mesh as close as those in the mesh before the refinement. Our choice is to project either elastic or plastic strain tensor based upon the additive decomposition of the total strain $\left(\boldsymbol{\epsilon}=\boldsymbol{\epsilon}^{e}+\boldsymbol{\epsilon}^{p}\right)$. As the total strains are recovered by the symmetric gradient of the displacement fields, which are always nodal values, the projection of plastic strain tensors result in the elastic strain tensors and vice versa. Once obtaining the strain values, the current stress state is recovered followed by $\boldsymbol{\sigma}=\mathbb{C}^{e}: \boldsymbol{\epsilon}^{e}=\mathbb{C}^{e}:\left(\boldsymbol{\epsilon}-\boldsymbol{\epsilon}^{p}\right)$, where $\mathbb{C}^{e}$ is the fourth-order elastic modulus [27]. The recovery of scalar and tensor variables including prime fields is described in the preceding section (Section 3.2). To sum up, the mesh refinement process is summarized as follows:

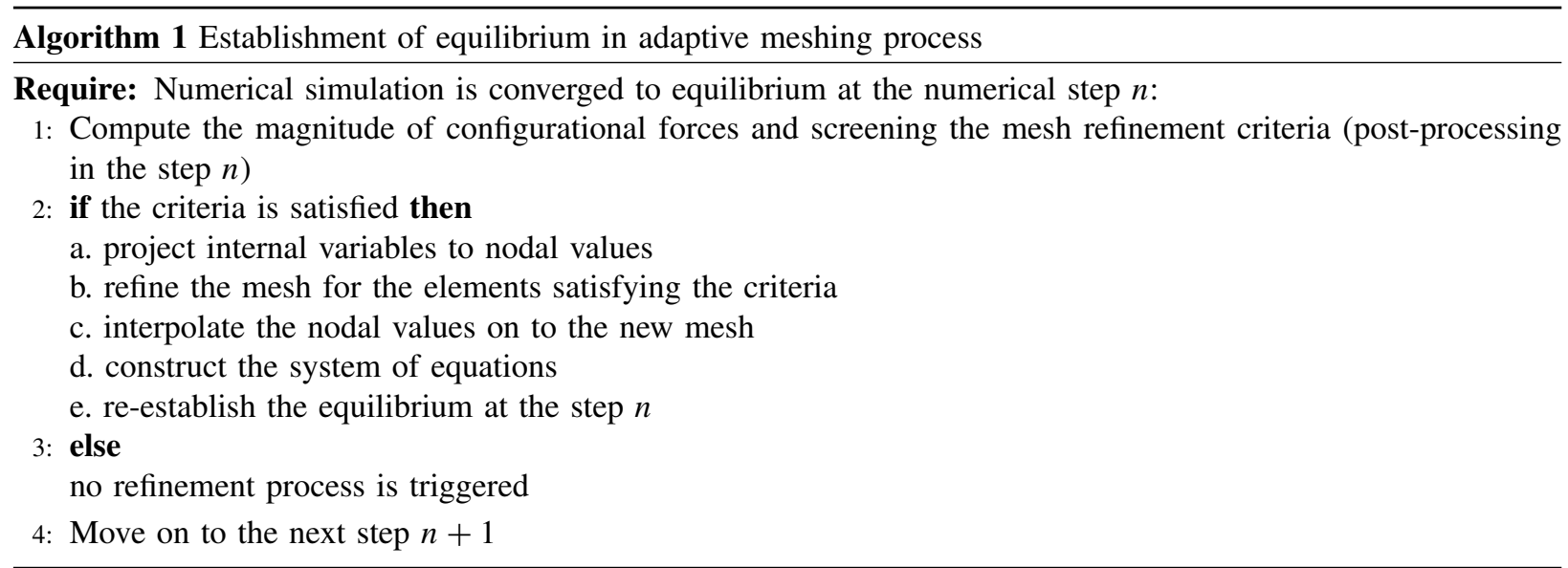

\section{Balance principles}

In this section we present balance principles and their finite element formulations for fluid-infiltrating materials associated with the micromorphic regularization. Started with the coupled equations for fluid-saturated porous media (e.g., [30]) excluding any geometric nonlinearity effect, the micromorphic balance equation is considered as an additional governing equation in order to regularize the given systems. For completeness, we briefly review the governing equations first. We then describe spatial and temporal discretizations followed by the solution strategy to resolve a system of nonlinear equations.

\subsection{Governing equations}

In this work, we employ a u-p formulation for the saturated poromechanics problems in the infinitesimal regime, which includes the momentum and mass balance equations $[79,80]$. Due to the nonlocal effect introduced into the constitutive law, the modified Helmholtz equation is added as an additional governing equation. Therefore, we may write the local forms of the balance equations as,

$$
\nabla \cdot\left(\boldsymbol{\sigma}^{\prime}-p \boldsymbol{I}\right)+\rho \boldsymbol{g}=\mathbf{0} \text { (Momentum balance) }
$$




$$
\begin{aligned}
& \nabla \cdot \dot{\boldsymbol{u}}+\nabla \cdot\left(-\frac{1}{\mu} \boldsymbol{k} \cdot \nabla p\right)=0 \quad \text { (Mass balance), } \\
& \alpha-l_{p}^{2} \Delta \alpha=\bar{\alpha} \quad \text { (Micromorphic balance). }
\end{aligned}
$$

Here, $\boldsymbol{\sigma}^{\prime}$ is effective Cauchy stress tensor, $p$ is pore pressure field, $\boldsymbol{I}$ is the second-order identity tensor, $\boldsymbol{u}$ is displacement field for the solid matrix, $\rho$ is the buoyant density of the mixture in the saturating fluid, $\boldsymbol{g}$ is the gravity acceleration vector, $\boldsymbol{k}$ is the effective permeability, $\mu$ ids the fluid viscosity, and $g$ is the magnitude of gravity acceleration vector $\boldsymbol{g}$, that is, $g=|\boldsymbol{g}|$. As described in the preceding section, $\alpha$ and $\bar{\alpha}$ indicate the global micromorphic and the local internal variables, respectively (Section 2).

Here we consider a domain $\mathcal{B}$ with boundary $\partial B$. This boundary is composed of Dirichlet boundaries (solid displacement $\partial \mathcal{B}_{u}$, fluid pressure $\partial \mathcal{B}_{p}$ ) and von Neumann boundaries (solid traction $\partial \mathcal{B}_{t}$, fluid flux $\partial \mathcal{B}_{q}$, micromorphic variable) as,

$$
\left\{\begin{array}{l}
\partial \mathcal{B}=\partial \mathcal{B}_{\boldsymbol{u}} \cup \partial \mathcal{B}_{t}=\partial \mathcal{B}_{p} \cup \partial \mathcal{B}_{\boldsymbol{q}} \\
\varnothing=\partial \mathcal{B}_{\boldsymbol{u}} \cap \partial \mathcal{B}_{\boldsymbol{t}}=\partial \mathcal{B}_{p} \cap \partial \mathcal{B}_{\boldsymbol{q}} .
\end{array}\right.
$$

The prescribed boundary conditions (Dirichlet and von Neumann boundary conditions) including the micromorphic boundary condition (11) read,

$$
\left\{\begin{array}{rll}
\boldsymbol{u}=\overline{\boldsymbol{u}} & \text { on } & \partial \mathcal{B}_{\boldsymbol{u}}, \\
\boldsymbol{\sigma} \cdot \boldsymbol{n}=\overline{\boldsymbol{t}} & \text { on } & \partial \mathcal{B}_{t}, \\
p=\bar{p} & \text { on } & \partial \mathcal{B}_{p}, \\
-\boldsymbol{n} \cdot \boldsymbol{q}=\bar{q} & \text { on } & \partial \mathcal{B}_{q}, \\
\nabla \alpha \cdot \boldsymbol{n}=0 & \text { on } & \partial \mathcal{B},
\end{array}\right.
$$

where $\boldsymbol{n}$ is outward unit normal on surface $\partial \mathcal{B}$. For model closure, the initial conditions are imposed as,

$$
\boldsymbol{u}=\boldsymbol{u}_{0}, \quad p=p_{0}, \quad \alpha=\alpha_{0} \text { at } t=t_{0} .
$$

We note that $\alpha_{0}$ is assumed to the preconsolidation pressure $p_{c}^{\prime}$ for the initial setting.

\subsection{Variational form}

We present the weak form of the given boundary value problem for the finite element implementation. First, the trial spaces for the weak form read,

$$
\begin{aligned}
V_{\boldsymbol{u}} & =\left\{\boldsymbol{u}: \mathcal{B} \rightarrow \mathbb{R}^{3}\left|\boldsymbol{u} \in\left[H^{1}(\mathcal{B})\right], \boldsymbol{u}\right|_{\partial \mathcal{B}_{u}}=\overline{\boldsymbol{u}}\right\}, \\
V_{p} & =\left\{p: \mathcal{B} \rightarrow \mathbb{R}\left|p \in H^{1}(\mathcal{B}), p\right|_{\partial \mathcal{B}_{p}}=\bar{p}\right\}, \\
V_{\alpha} & =\left\{\alpha: \mathcal{B} \rightarrow \mathbb{R} \mid \alpha \in H^{1}(\mathcal{B})\right\} .
\end{aligned}
$$

Here $H^{1}$ denotes the Sobolev space of degree one. The corresponding admissible spaces of variations are defined as,

$$
\begin{aligned}
V_{\boldsymbol{\eta}} & =\left\{\boldsymbol{\eta}: \mathcal{B} \rightarrow \mathbb{R}^{3}\left|\boldsymbol{\eta} \in\left[H^{1}(\mathcal{B})\right], \boldsymbol{\eta}\right|_{\partial \mathcal{B}_{u}}=\mathbf{0}\right\}, \\
V_{\psi} & =\left\{\psi: \mathcal{B} \rightarrow \mathbb{R}\left|\psi \in H^{1}(\mathcal{B}), \psi\right|_{\partial \mathcal{B}_{p}}=0\right\}, \\
V_{\phi} & =\left\{\phi: \mathcal{B} \rightarrow \mathbb{R} \mid \phi \in H^{1}(\mathcal{B})\right\} .
\end{aligned}
$$

The weak form of the problem is then to find $\{\boldsymbol{u}, p, \alpha\} \in V_{\boldsymbol{u}} \times V_{p} \times V_{\alpha}$ such that for all $\{\boldsymbol{\eta}, \psi, \phi\} \in V_{\eta} \times V_{\psi} \times V_{\phi}$,

$$
G(\boldsymbol{u}, p, \alpha, \boldsymbol{\eta})=H(\boldsymbol{u}, p, \alpha, \psi)=L(\boldsymbol{u}, p, \alpha, \phi)=0,
$$

where $G: V_{\boldsymbol{u}} \times V_{p} \times V_{\alpha} \times V_{\eta} \rightarrow \mathbb{R}$ is the weak statement of linear momentum balance, that is,

$$
G(\boldsymbol{u}, p, \alpha, \boldsymbol{\eta})=\int_{\mathcal{B}}\left[\nabla^{s} \boldsymbol{\eta}: \boldsymbol{\sigma}^{\prime}-p \nabla \cdot \boldsymbol{\eta}-\boldsymbol{\eta} \cdot \rho \boldsymbol{g}\right] d V-\int_{\partial \mathcal{B}_{t}} \boldsymbol{\eta} \cdot \overline{\boldsymbol{t}} d \Gamma .
$$


$H: V_{\boldsymbol{u}} \times V_{p} \times V_{\alpha} \times V_{\psi} \rightarrow \mathbb{R}$ is the weak statement of mass balance, that is,

$$
H(\boldsymbol{u}, p, \alpha, \psi)=\int_{\mathcal{B}} \psi \nabla \cdot \dot{\boldsymbol{u}} d V-\int_{\mathcal{B}} \nabla \psi \cdot\left(-\frac{1}{\mu} \boldsymbol{k} \cdot \nabla p\right) d V-\int_{\partial_{\mathcal{B}_{q}}} \psi \bar{q} d \Gamma .
$$

And $L: V_{\boldsymbol{u}} \times V_{p} \times V_{\alpha} \times V_{\phi} \rightarrow \mathbb{R}$ is the weak statement of micromorphic balance, that is,

$$
L(\boldsymbol{u}, p, \alpha, \phi)=\int_{\mathcal{B}} \phi(\alpha-\bar{\alpha}) d V+\int_{\mathcal{B}} \nabla \phi \cdot\left(l_{p}^{2} \nabla \alpha\right) d V .
$$

Considering the time-dependent nature of mass balance equation (49), the weak form is discretized in time. For implementation, we introduce a temporal discretization using the backward Euler scheme. Temporal discretizations and their linearization are straightforward, which are not described in this work for brevity. Interested readers please refer to Borja [81], White and Borja [30], Sun [82], Choo and Borja [83], Sun et al. [84], Na and Sun [29] for details.

\subsection{Operator-split solver and solution strategies}

In this work, the standard Galerkin form of the governing equations are discretized using only one set of basis functions. Note that, due to the lack of the inf-sup condition, the standard u-p formulation may lead to spurious responses. For brevity, the details of the stabilization procedure is not discussed in this work. Interested readers please refer to Zienkiewicz et al. [85], Preisig and Prévost [86], White and Borja [30], Sun et al. [84,87], Wang and Sun [16,88] for details. The operator-split solution strategy is adopted to solve the problem. To be specific, the momentum and mass balance equations are advanced monolithically followed by the micromorphic regularization equation. This approach simplifies the derivation of the local Jacobian for Newton's iteration and the global consistent linearization procedure (cf., [89-91]). Therefore, the finite element discretization of (35) to (37) and the corresponding linearization are straightforward because no couplings need to be constructed under the micromorphic setting. However, the time step (or load increment) to solve the problem with the operator-split approach requires the time step (or load increment) to be small enough to achieve sufficient accuracy. As the reestablishment of equilibrium step after the mesh refinement leverages the converged solution before refinement, the accuracy and stability in the converged solution is an integral part. Interested readers may refer to previous work [24,92-94] for more discussions on this aspect. Note that the equal-order finite element approach is adopted, where we use the stabilization scheme to resolve inf-sup deficiency under undrained condition by White and Borja [30].

\section{Numerical examples}

We present numerical examples to show the performance of the proposed remeshing algorithm associated with the gradient-enhanced anisotropic Cam-Clay models. Firstly, we design 2D plane compression tests in which the mechanical and hydraulic responses are obtained by changing the bedding plane orientations. We further analyze the influence of the nonlocal micromorphic energy $\left(\phi_{m}\right.$ in (17)) on the configurational force associated with mesh refinement. Second, we investigate how the configurational force criteria that are established by different poromechanics assumptions (Section: 3.1.2) affect the numerical simulations when the concentrated strain localization zone (as in the cases of shear banding) and sharp pore pressure gradient (as in the cases of injection or extraction in porous media of low effective permeability) exist. In the last numerical example, the number of mesh refinement and associated configurational force changes are investigated, which shows how the configurational force are used as refinement criteria. The validation of the local anisotropic Cam-Clay model with experimental results, the computational efficiency of adaptive meshing simulations, and the performance of micromorphic regularization for mesh independence are included in Appendices A, B, and C, respectively.

\subsection{Effect of anisotropy and nonlocal micromorphic energy}

We firstly demonstrate how the adaptive mesh-refinement helps to capture the strain localization in anisotropic numerical specimens. Fig. 2 presents schematics of the 2D plane strain compression test with the geometry dimensions of $B=0.05 \mathrm{~mm}$ and $L=0.1 \mathrm{~mm}$ and the boundary conditions. Note that all boundaries are impervious except that the top surface of the specimen is drained. The material parameters are selected to approximate 


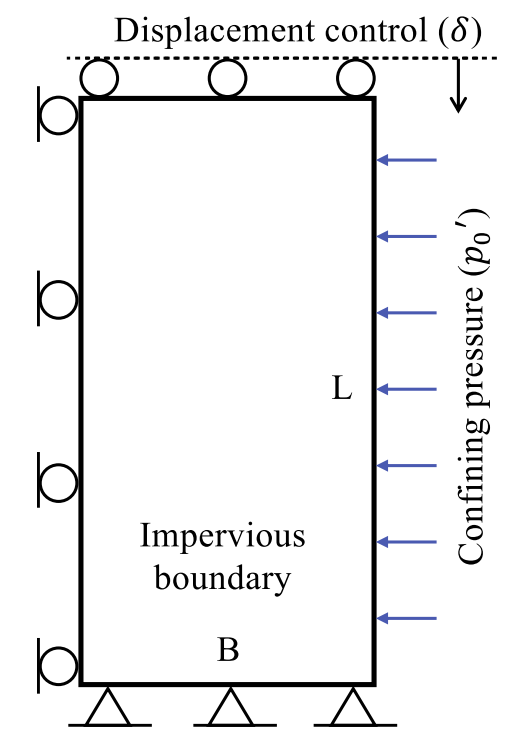

Fig. 2. Schematics of the biaxial compression problem: the geometry, boundary, and loading conditions.

Tournemire shale (cf., [22,95]), in which the elasticity parameters are assumed as $E_{1}=24,000 \mathrm{MPa}, E_{2}=14,000$ $\mathrm{MPa}, v_{12}=0.3, v_{23}=0.25$; the plasticity parameters are assumed as $\lambda_{p}=0.0026 ; M=1.19 ; \alpha^{\mathrm{p}}=1.25$; $\beta^{\mathrm{p}}=0.95 ; \gamma^{\mathrm{p}}=1.0$; the micromorphic regularization parameters are assumed as $l_{p}=1.0 \times 10^{-2} \mathrm{~mm}$ and $\varepsilon_{p}=10.0$. We note that the plastic length scale $l_{p}$ is selected in the order of mesh size for regularization purposes via the micromorphic setting. This parameter, however, can be used to characterize size dependent attributes of materials, such as the width of localized plastic deformation, fracture, fabrics, and slip systems associated with the experimental observation or the microscopic features. In the micromorphic regularization approach, the penalty term $\varepsilon_{p}$ links the local $(\bar{\alpha})$ and global $(\alpha)$ internal variables in the hardening equation, in which they become equivalent when $\varepsilon_{p}$ is high enough [20]. For each simulation, $\varepsilon_{p}$ is determined by preliminary tests such that it properly regularizes the problem under the softening behavior.

The hydraulic parameters are assumed to be isotropic with the intrinsic permeability and viscosity of $1.0 \times 10^{-15}$ $\mathrm{m}^{2}$ and $1.0 \times 10^{-6} \mathrm{kPa}$ s, respectively. The initial preconsolidation pressure $p_{c_{0}}^{\prime}$ is set to $-100 \mathrm{MPa}$ while the initial confining pressure $p_{0}^{\prime}$ of $-40 \mathrm{MPa}$ is used. We then select three normal vectors to the plane of isotropy in 2D conditions, which are rotated $0^{\circ}\left(\theta=0^{\circ}\right), 45^{\circ}\left(\theta=45^{\circ}\right)$, and $90^{\circ}\left(\theta=90^{\circ}\right)$ from the horizontal axis (i.e., x-axis), respectively. For example, the normal vector is approximately vertical for many in-situ layers of geomaterials, which has a rotation of $90^{\circ}$.

Fig. 3 shows a collection of global stress-strain curves measured at the top of the specimen with different orientations of the plane of the isotropy. As the material is set to be transversely isotropic, different stress-strain curves are obtained when the bedding plane is rotated. The influence of anisotropy associated with the mesh refinement is further presented in Fig. 4. As the anisotropy of the plastic behavior only manifests in the deviatoric component of stress for this type of model [22], the deviatoric strain distribution is shown. Furthermore, the pore pressure patterns are plotted to demonstrate the local fluid flow due to the hydro-mechanical coupling.

Due to the fact that the bottom of the boundary is fixed, the numerical specimen cannot maintain a homogeneous stress state throughout the simulations. This heterogeneous stress state (1) leads to the shear bands initiated at the lower right corner where the pin boundary condition ends and (2) affects the orientation of the resultant shear bands such that they may misalign with the principal direction of the acoustic tensor associated with the zero eigenvalue [96].

When $\theta=0^{\circ}$, no distinct strain localization has been observed (Fig. 4(a)). As the bedding planes are horizontally aligned, the weak plane also lead to a lower peak shear strength and a more diffusive strain distribution. The influence of horizontal bedding can also be identified from the pore pressure distribution in Fig. 4(a), in which the excess pore pressure is developed around the bottom region of the specimen while the drained top condition is 


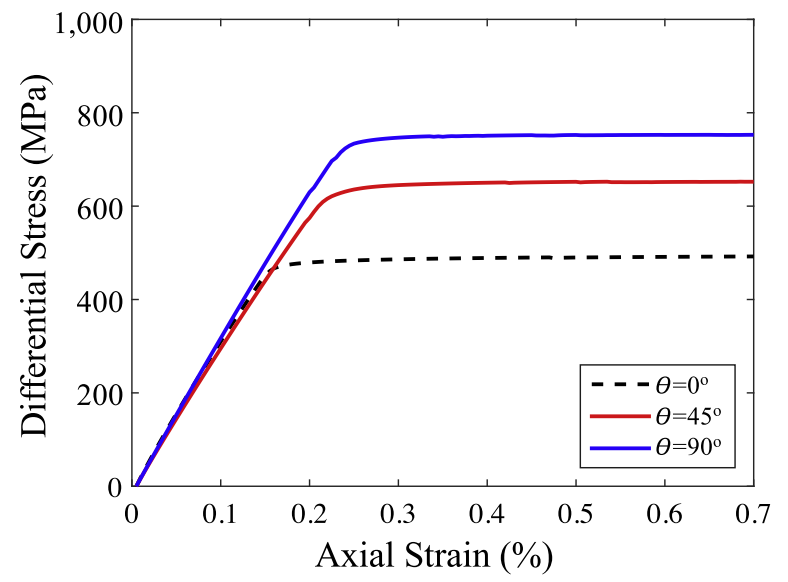

Fig. 3. Collection of stress-strain curves measured at the top of the specimen with the different orientations of the plane of isotropy in $2 \mathrm{D}$ conditions $\left(\theta=0^{\circ}, \theta=45^{\circ}\right.$, and $\theta=90^{\circ}$, respectively).
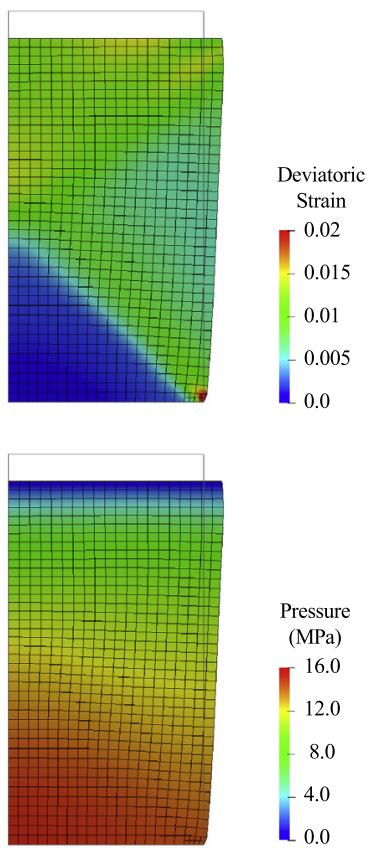

(a) $\theta=0^{\circ}$
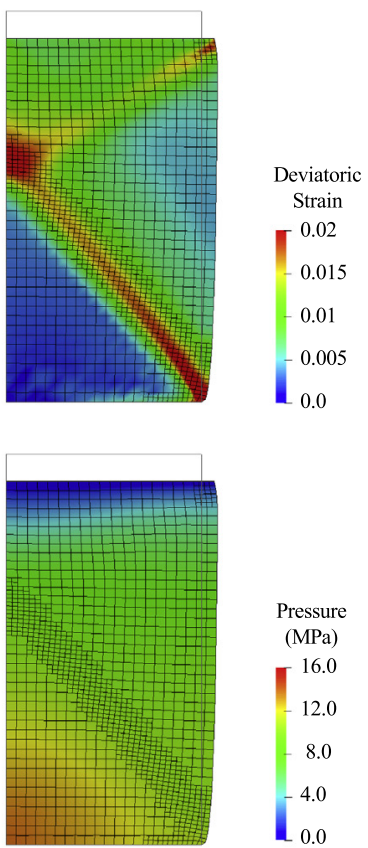

(b) $\theta=45^{\circ}$
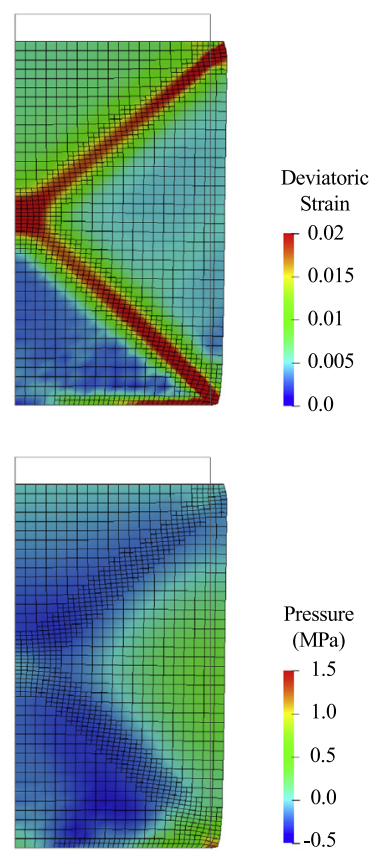

(c) $\theta=90^{\circ}$

Fig. 4. Patterns of deviatoric strain and pore pressure when the orientations of the bedding plane are: $\theta=0^{\circ}, \theta=45^{\circ}$, and $\theta=90^{\circ}$, respectively (adaptive mesh-refinement is activated).

adopted. As the bedding plane is aligned with the diagonal directions $\left(\theta=45^{\circ}\right)$, the strain localization is captured inside the region with mesh refinement (Fig. 4(b)). More important, we obtain that the configurational force criterion is also able to determine the demand to refine the mesh near the strain localization where the gradient of the displacement field is the sharpest in the spatial domain. Despite of the two-way hydro-mechanical coupling, this solid skeleton configuration force does not trigger any mesh refinement at the top region of the numerical specimen where the pore pressure gradient is most significant.

On the other hand, when the bedding plane is aligned vertically $\left(\theta=90^{\circ}\right)$, two distinct shear bands are formed where the excess pore water pressure is dissipated quickly than in the previous two cases (Fig. 4(c)). According to Semnani et al. [22], Tien et al. [97], one dominant shear band is likely to be observed when the bedding planes 

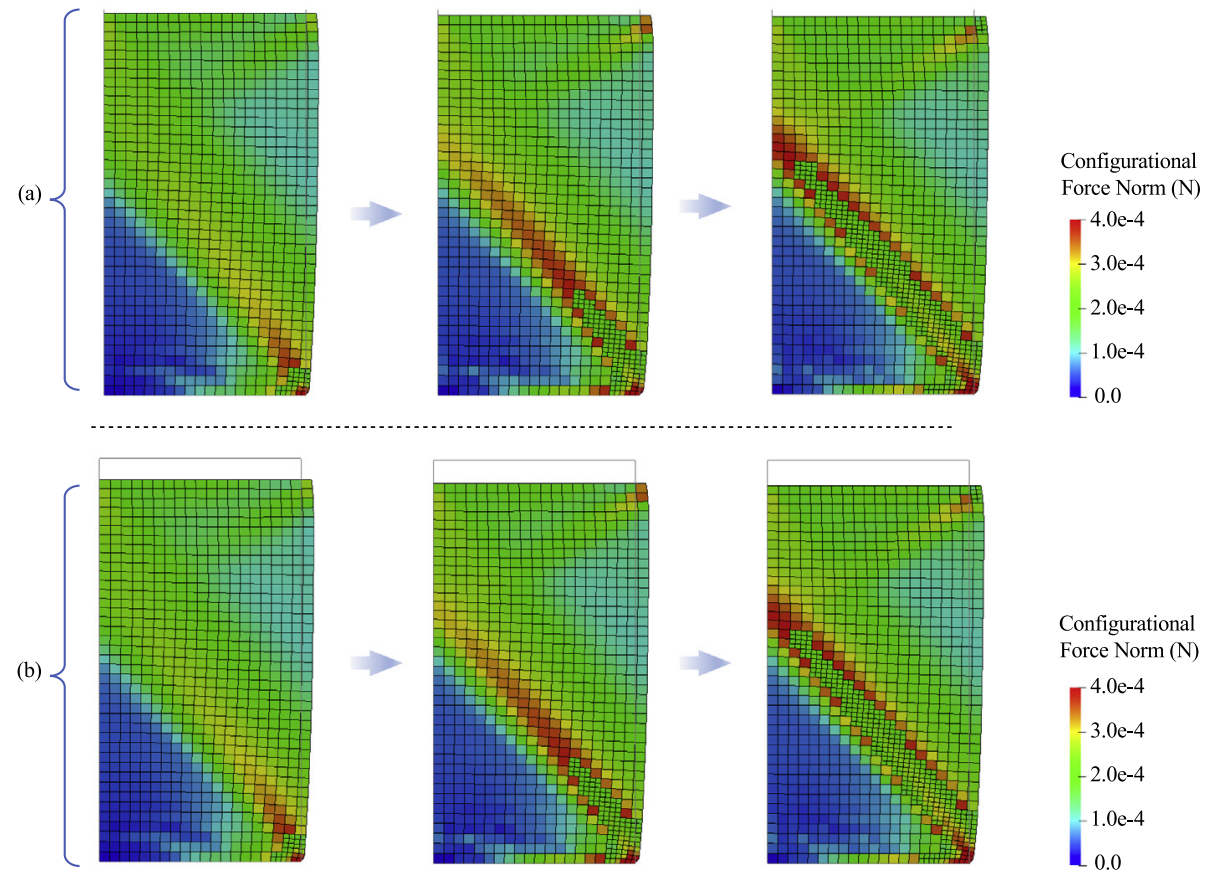

Fig. 5. Sequence of adaptive-mesh refinement with the patterns of configurational force magnitude to identify the influence of nonlocal micromorphic energy on refinement criteria: (a) when the strain energy (solid part) is only considered; (b) when both local strain energy and nonlocal micromorphic energy are considered.

are aligned neither horizontally or vertically. In other words, when the bedding plane is aligned either horizontally or vertically $\left(\theta=0^{\circ}\right.$ or $\left.90^{\circ}\right)$, no shear band or multiple shear bands are observed in the simulations. When the bedding plane is $\theta=45^{\circ}$ in Fig. 4(b), however, one dominant shear band is observed, which confirms the phenomenon stated in $[22,97]$.

Secondly, we investigate the influence of nonlocal micromorphic energy on configurational forces for mesh refinement. Note again the results in Fig. 4 only use the solid part in energy-momentum tensor (25) to determine the configurational force. In Fig. 5(a), the magnitude of configurational forces are depicted along with the loading sequence and associated mesh refinement, where the refinement criterion is set to: $G_{c r i t}^{I}=4.0 \times 10^{-4} \mathrm{~N}$. Only the case when the bedding orientation $\theta=45^{\circ}$ is presented for comparison. Under the same numerical conditions, the nonlocal micromorphic energy $\phi_{m}$ is added to the energy-momentum tensor $\Sigma$ in (25), which results in Fig. 5(b). Knowing that the nonlocal micromorphic energy term is highly related to the plastic regions, we expect that this term may be a good indicator by increasing the configurational force around that region. As can be seen in Fig. 5(a) and (b), however, the influence of the nonlocal micromorphic energy terms on configurational force is hardly observed. Although not explicitly shown in Fig. 5, the magnitude of nonlocal micromorphic energy terms $\left(\phi_{m}\right)$ is observed to be less than $0.1 \%$ of the magnitude of the free energy of the solid skeleton. This indicates that (1) the operator-split strategy is able to suppress the difference between the local and micromorphic internal variables such that $\alpha-\bar{\alpha}$ is sufficiently small, and (2) the Laplacian term of the micromorphic internal variable generates only insignificant amount of configurational force compared to those from the elastic stored energy and the local plastic work. Presumably, this phenomenon is attributed to the physical length scale and the penalization parameters $l_{p}$ and $\varepsilon_{p}$ in (37) selected for this simulation. A different set of material parameters may alter the result. Nevertheless, by comparing the benchmark and the adaptive simulations, such as the results shown in Fig. 20, does indicate that the gradient dependence introduced via the nonlocal energy functional is able to circumvent the mesh dependence.

\subsection{Configurational poromechanics and anisotropy in flow}

We test different refinement criteria for the multiphysical poromechanics problems described in Section 3.1. In the previous numerical example, we showcase the hydro-mechanical responses driven by mechanical loading. In this 


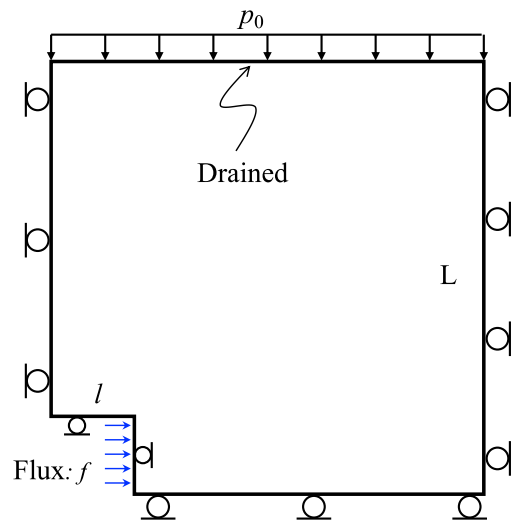

(a)

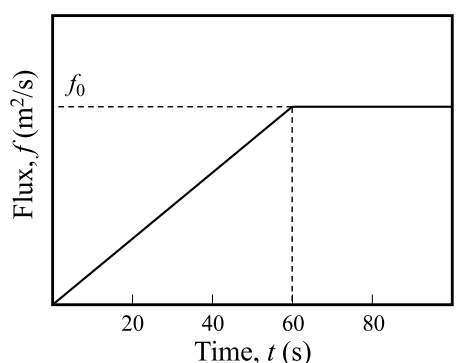

(b)

Fig. 6. Schematics configurational poromechanics.

example, we design an injection well problem to examine how the configurational-force refinement criteria perform when the external loading is hydraulic driven. The schematics of the numerical domain and boundary conditions are presented in Fig. 6(a). The geometry with the injection well has dimensions of $L=0.1 \mathrm{~m}$ and $l=0.01 \mathrm{~m}$. The drained boundary is assumed only at the top surface where no displacement constraint is applied. The water injection is approximated by applying flux boundary $f$, that is controlled as in Fig. 6(b). The displacement constraint on other boundaries are also depicted in Fig. 6(a), in which the horizontal direction on the injection surface is constrained as well to limit excessive deformation due to high fluid injection. The material properties are assumed to be isotropic to streamline the test scenarios and limit the mechanical anisotropic influence. Therefore, the elastic parameters are set to $E=2,400 \mathrm{kPa}$ and $v=0.3$; the plastic parameters are assumed as $\lambda_{p}=0.01 ; M=1.0 ; p_{c_{0}}^{\prime}=100 \mathrm{kPa}$, and $p_{0}^{\prime}=80 \mathrm{kPa}$. The micromorphic regularization parameters are selected as $l_{p}=5.0 \times 10^{-3} \mathrm{~m}$ and $\varepsilon_{p}=10.0$.

Firstly, the hydraulic parameters are assumed to be isotropic, in which the intrinsic permeability and viscosity are assumed as $1.0 \times 10^{-12} \mathrm{~m}^{2}$ and $1.0 \times 10^{-6} \mathrm{kPa}$.s, respectively. We then evaluate the configurational force with different assumptions followed by Section 3.1.2, that is, Case A considers the effective stress under the drained limit condition (25); Case B considers the total stress under the undrained limit assumption (27); Case C considers the pressure dissipation in addition to the total stress (28). Using these different configurational forces, numerical simulations are conducted and their results are presented in Fig. 7. Although the configurational forces are calculated associated with different assumptions, the same magnitude is set for the refinement criteria: $G_{c r i t}^{I}=5.0 \times 10^{-3} \mathrm{kN}$. When we consider the solid part only and use the effective stress for configurational force calculation (Case A), the mesh was refined only near the injection well edge. When we take the total stress and dissipation (Cases B and C), however, the mesh refinement regions are expanded associated with the pressure. Furthermore, the refined region are shown to be similar with the displacement patterns because the hydro-mechanical coupling term also exists in configurational force calculation (27). While the refinement criteria for Cases B and C show the same results to that of Case A under the biaxial compression tests (the mechanical loading is applied), the two Cases give rise to distinct difference when the fluid flow is highlighted in poromechanics problems.

Next, we introduce anisotropy in the intrinsic permeability to investigate how the configurational force approach performs in anisotropic fluid-infiltrating porous media. The transversely isotropic permeability is approximated as in Fig. 7 so that sharp pressure gradients are developed in different regions of the domain. In other words, the same boundary conditions are used as in Fig. 6 while several layers of lower permeability are embedded horizontally. We purposely design this layered permeability domain to clearly identify its influence on mesh refinement associated with different configurational force criteria. The influence of lower permeability layers on pressure development and mesh refinement are presented in Fig. 8 for Cases A, B, and C, respectively. Although the higher pressure are observed due to the lower permeability layers around the injection well, less mesh-refinement is activated in Case A. For Cases B and C, however, the refined regions are expanded along the bottom layer. Although the minor difference is observed with respect to the mesh-refinement from Cases B and C except its growing region circled 

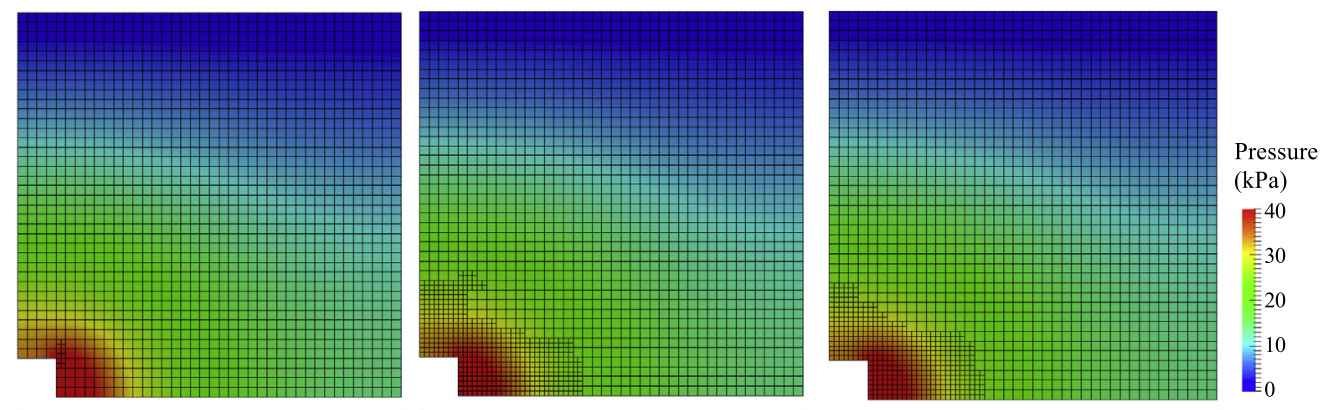

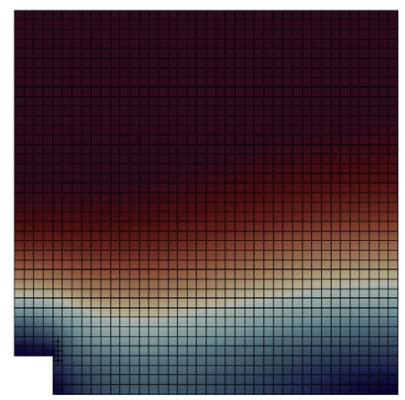

Case A

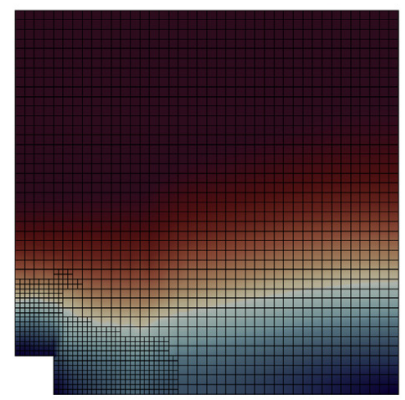

Case B

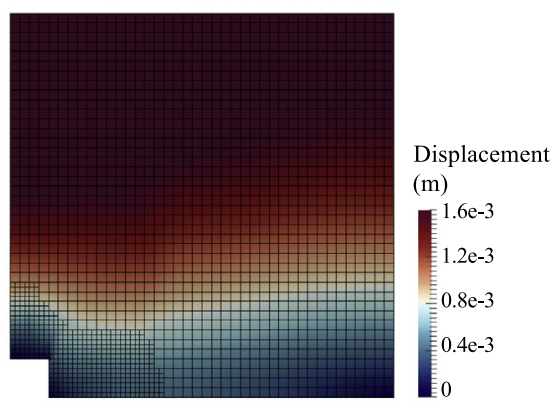

Case C

Fig. 7. Patterns of pressure and displacement distribution with adaptive mesh refinement based on different configurational force calculations: Case A(Effective stress), Case B(Total stress), and Case C (Total stress + Dissipation) - Isotropic material properties.

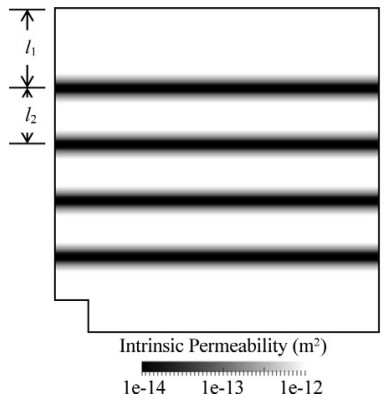

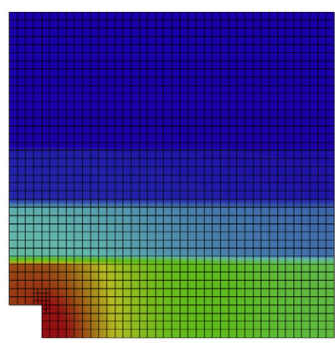

Case A

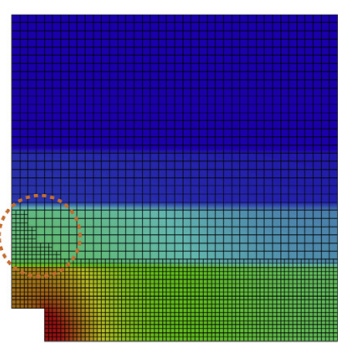

Case B

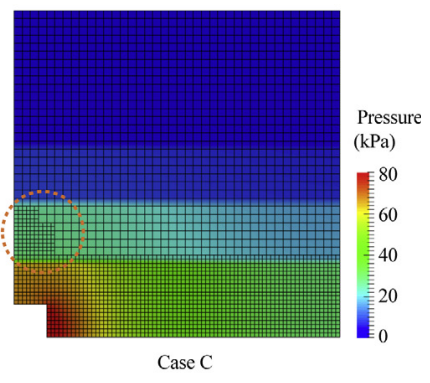

Fig. 8. Approximated transversely isotropic permeability distribution (Left) and pressure patterns with adaptive mesh refinement based on different configurational force calculations: Case A(Effective stress), Case B(Total stress), and Case C (Total stress + Dissipation).

in Fig. 8, this example shows that the configurational force can be used as a good indicator of mesh-refinement for flow dominant problems when the poromechanics theory is introduced.

To investigate how the refinement evolves along with pressure development for Cases B and C, we present their successive results in Fig. 9. When the flux was initiated, the refinement region is wider in Case C compared to Case B (Fig. 9(a)). As the pressure increases due to the flux boundary condition, the refined region in Case $\mathrm{C}$ is progressed slightly ahead of that of Case B. This tendency continues along with the simulations while the overall results from Cases B and C may not see much difference. Because the dissipation term $\left(\mathcal{D}_{f}\right)$ in $(17)$ is closely related to the pressure gradient, the frontier where the pressure changes drastically due to flux boundary condition is refined repeatedly until the flow reaches to steady state.

The magnitude of configurational force measured by (a) effective stress ( $\left.W_{\text {solid }}\right)$, (b) total stress $\left(W_{\text {solid }}+W_{\text {fluid }}\right)$, and $(c)$ dissipation $\left(\mathcal{D}_{f}\right)$ terms in $(28)$ are shown in Fig. 10. We purposely present contributions of each term followed by the method (29) for comparison. Fig. 10(a) of Case A, for example, shows the magnitude of configurational force by $W_{\text {solid, }}$, which is concentrated at the edge of the injection well. Similarly, Fig. 10(b) of Case A shows that the configurational force measured by $W_{\text {solid }}+W_{\text {fluid }}$ are accumulated around the injection well due to the pressure boundary condition. However, the mesh was not refined because only the contribution by $W_{\text {solid }}$ 

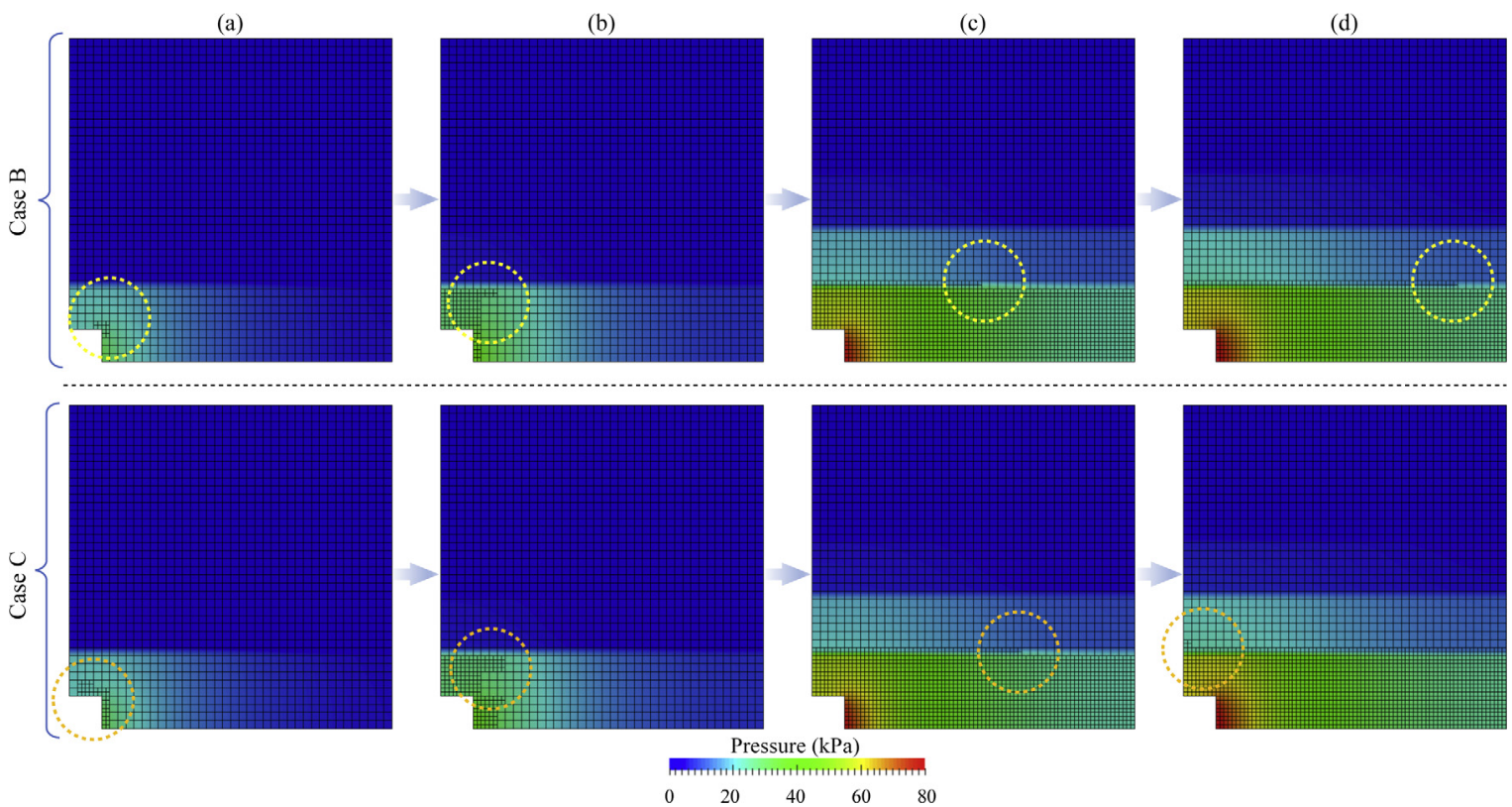

Fig. 9. Successive mesh refinement results along with the pressure development when the refinement criteria is based on Cases B and C.

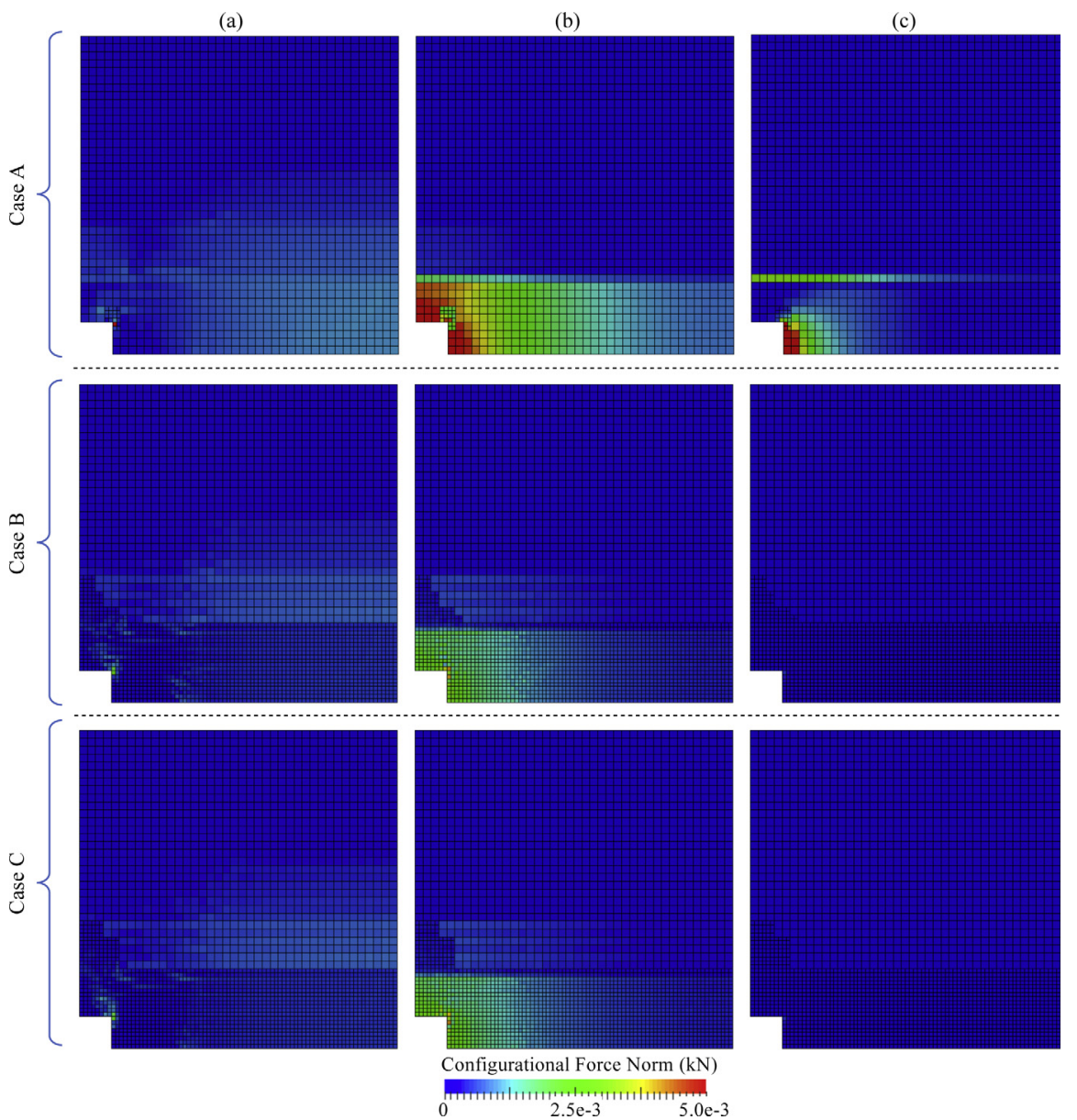

Fig. 10. Change of configurational force under Cases A, B, and C: (a) Effective stress; (b) Total stress; (c) Dissipation. 


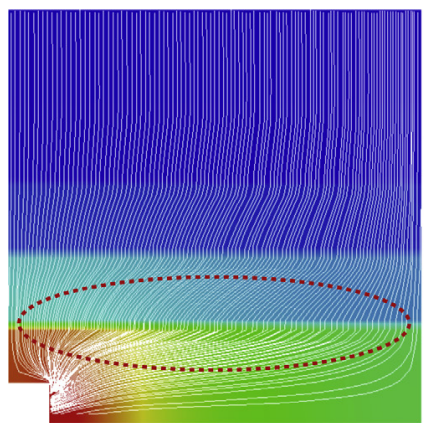

Case A

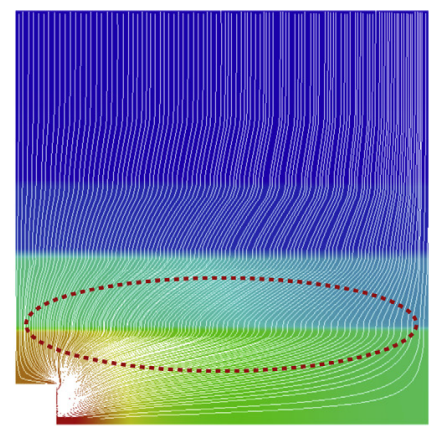

Case B

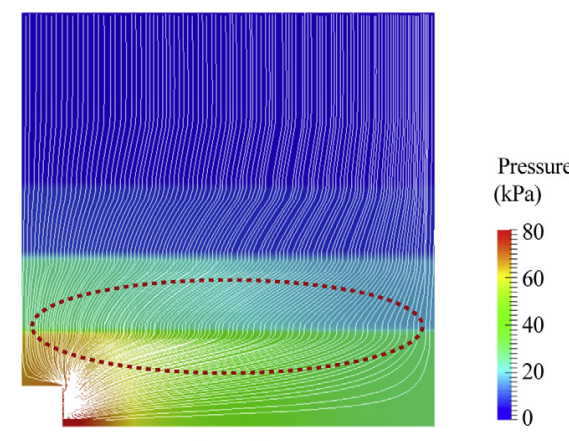

Case C

Fig. 11. Streamlines and pressure patterns for Cases A, B, and $C$ that shows improvement in capturing sharp changes of pressure gradient when the mesh was refined along the low permeability layers (circled).

is used as refinement criterion for Case A. Likewise in Fig. 10(c) of Case A, the magnitude of configurational force due to $\mathcal{D}_{f}$ is presented, which indicates the regions where sharp pressure gradient is expected (injection well surface and low permeability layer). Therefore, in Fig. 10(a), (b), and (c) of Case A, we can identify the patterns of accumulated configurational force by $W_{\text {solid }}, W_{\text {solid }}+W_{\text {fluid }}$, and respectively. In other words, the contributions of $W_{\text {solid }}+W_{\text {fluid }}$ and $\mathcal{D}_{f}$ can be used to indicate the regions where sharp pressure changes are occurred. Cases $\mathrm{B}$ and $\mathrm{C}$ in 10, finally, show how the configurational forces are dissipated due to mesh refinement. While $\mathcal{D}_{f}$ is quickly reduced along with refinement, $W_{\text {solid }}+W_{\text {fluid }}$ still exists. As the refined regions observed from Cases B and C showed little difference, we may further infer that the contributions by $W_{\text {solid }}+W_{\text {fluid }}$ are more dominant than $\mathcal{D}_{f}$ in the calculations of configuration force.

The advantages of using the mesh refinement in poromechanics problems can be found in Fig. 11, where the sharp changes of pressure gradient are better captured when the refined mesh are used. In other words, when we introduce promechanics theory into the configurational force associated with the adaptive meshing technique, we can not only capture the strain localization, but the sharp pressure gradient.

\subsection{Mesh refinement and configurational force}

In our final example, we introduce an idealized vertical cut problem, a common boundary value problem in geotechnical engineering, to test the capacity of the refinement criteria to handle the demanding cases that require consecutive mesh refinements. The mesh refinement as a configurational force dissipation mechanism is further examined and discussed in this set of numerical experiments. The schematics of the numerical test are depicted in Fig. 12. The dimension of the domain $L=10.0 \mathrm{~m}$, and the displacement control is used to apply vertical loading in $B(=5.0 \mathrm{~m})$. The domain in globally undrained, and the initial number of finite element used in the simulations is 400 . We adopt the constitutive model with isotropic setting for simplicity and assume the material parameters as in the second numerical example (Section 5.2) while the micromorphic regularization parameters are assumed as $l_{p}=0.05 \mathrm{~m}$ and $\varepsilon_{p}=0.1$. The initial preconsolidation pressure $p_{c}^{\prime}$ is set to $-1000 \mathrm{kPa}$, and no gravity acceleration is assumed. The effective volumetric pressures are purposely set to $-300 \mathrm{kPa}$ for establishing the initial equilibrium.

Given the fixed refinement criterion $G_{c r i t}^{I}=0.4 \mathrm{kN}$, we allow the mesh to be refined once and twice, respectively. In other words, the refined regions can be further discretized into the smaller elements provided the remeshing criterion is met. The magnitude of configurational force and the progress of mesh refinement are presented in Fig. 13. From Fig. 13(a), we can clearly identify that the mesh is refined as the configurational force increases, where its magnitude is dissipated, along with the refined region. On the other hand, the configurational forces are continuously accumulated inside the refined region due to the loading conditions we applied in this example. This region is where the strain localization is developed that forms the shear band. Fig. 13(b) presents how the multiple mesh refinement is performed. Due to high accumulation of configurational forces, the refined region is further discretized into smaller meshes which further dissipates the configurational force. The performance of the multiple mesh refinement is presented in Fig. 14(a). Regardless of the number of mesh refinements take place, the resultant total vertical reaction force obtained from these simulations does not exhibit significant difference. 


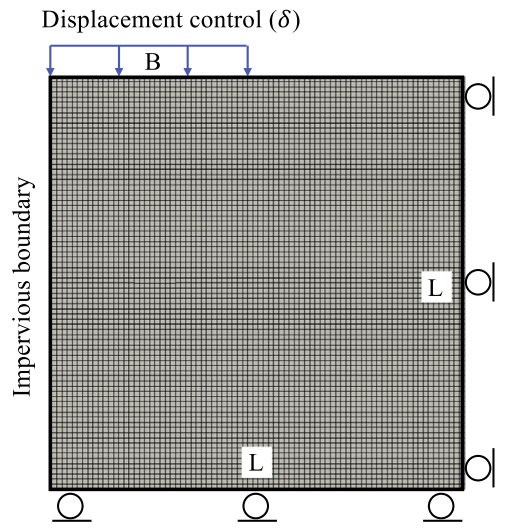

Fig. 12. Schematics of vertical cut problem with boundary conditions: all the boundaries are assumed undrained and the displacement control is used to apply loading.

(a)
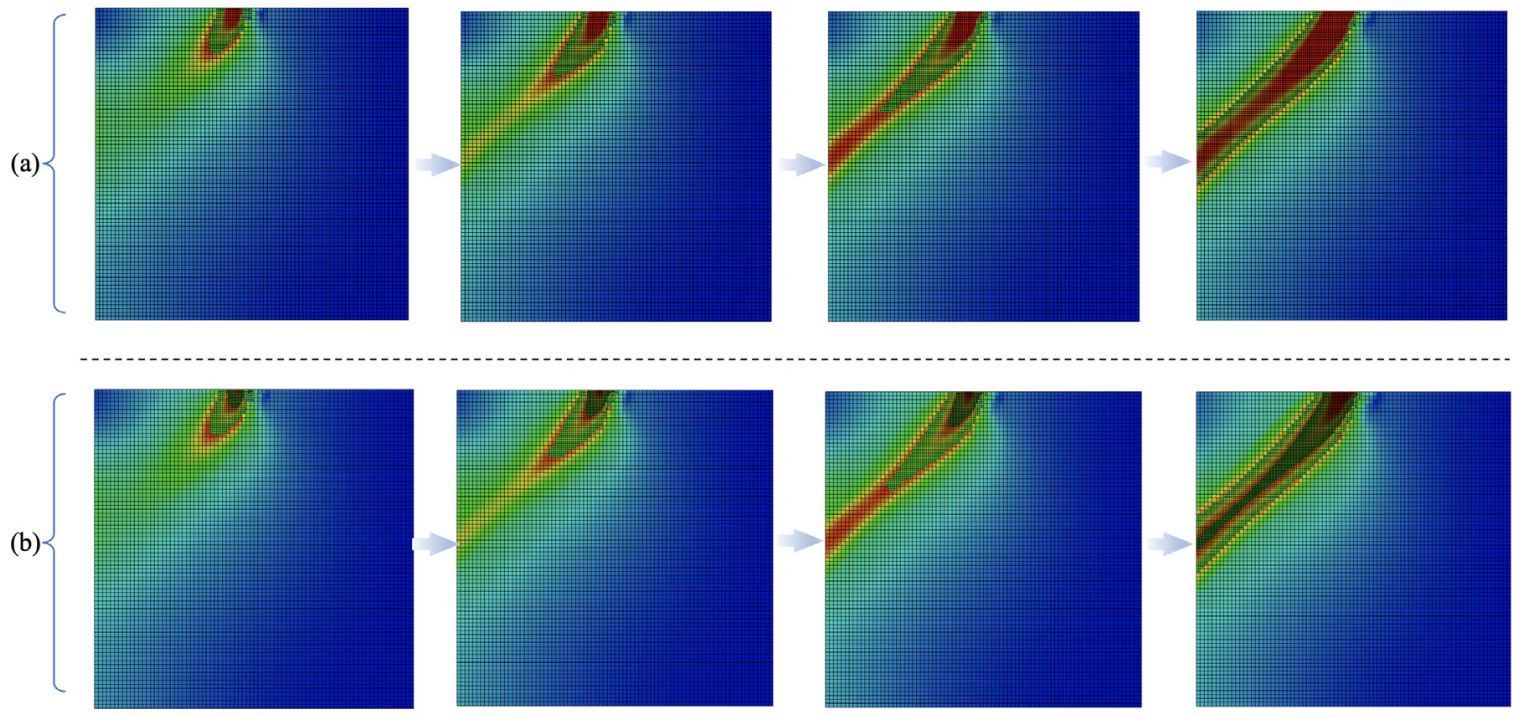

Configurational Force Norm $(\mathrm{kN})$

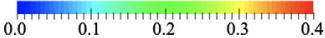

Fig. 13. Progress of mesh refinement and patterns of configurational force magnitude along with loading steps. The refinement is allowed once in (a) and twice in (b), respectively.

To investigate how the configurational force dissipates with respect to the number of mesh refinement, we select a specific point located inside the strain localization (Fig. 14(b)). In this location, the magnitude of configurational forces is measured and presented in Fig. 14(c). As expected, the configurational force is dissipated when the first mesh refinement is performed while it continues to increase over the refinement criterion when no more refinement is conducted. The horizontal line is depicted in Fig. 14(c) to indicate the mesh refinement criterion. When the refinement is performed twice, the configurational force is further dissipated and its magnitude is maintained below the refinement criterion.

\section{Conclusion}

We develop a mathematical framework that employs the micromorphic regularization to establish a nonlocal anisotropic critical state plasticity model. An adaptive mesh refinement procedure that employs configurational poromechanics theory to generate refinement criteria and Lie algebra mapping to deal with tensorial history variables 


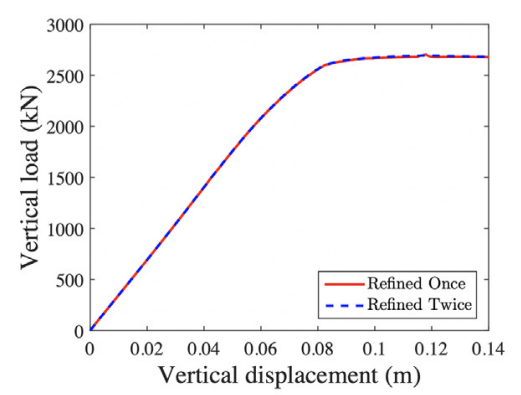

(a)

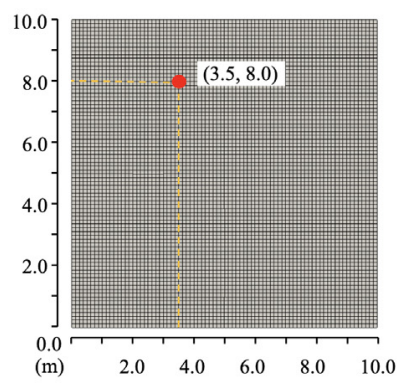

(b)

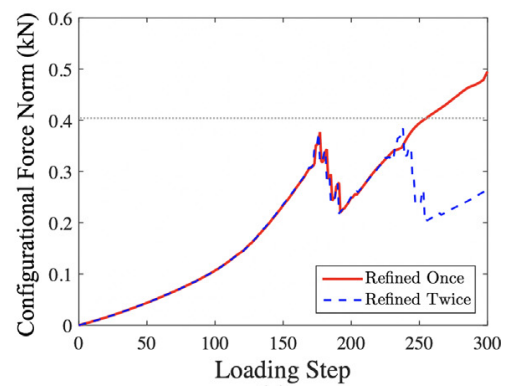

(c)

Fig. 14. Results when the mesh refinement is allowed once and twice, respectively, (a) the vertical load-displacements curves; (b) a location where the configurational force is measured and compared; (c) the magnitude of configurational force along the loading steps.

are established to resolve the sharp displacement and pore pressure gradients that may occur for the boundary value problems common in geotechnical and petroleum engineering and geological disposal.

Concepts from poromechanics theory (i.e., effective stress, total stress, and pressure dissipation) is incorporated in the derivation of the configurational force such that the resultant refinement criteria may account for hydromechanical coupling effect exhibited in fluid-infiltrating porous materials. By taking account of the contributions from the additional terms that lead to the gradient dependent plastic flow, we present a derivation of configurational force that is suitable to be used as a remeshing criterion for both two-phase Boltzmann continua and the higher-order counterparts.

In addition, a Lie algebra internal variable mapping is used such that the history-dependent behaviors for the new configuration can be captured in the new equilibrium state. A local-global operator-split algorithm is used to simplify the implementation. The numerical example indicates that the influence of nonlocal micromorphic energy is minor on configurational force calculations for mesh refinement. While the different configurational forces associated with poromechanics theory show little difference in performance when the mechanical loading is applied, the multi-physical configurational force on apparent solid skeleton is shown to be capable of triggering refinements that are necessary to capture the flow front and sharp pressure gradient. Finally, the vertical cut problem indicates that the proposed model yields consistent results regardless of the number of mesh refinement. These numerical experiments demonstrate that the configurational force can be used as a unified refinement criterion that handles a wide spectrum of situations, such as those involving Boltzmann or high-order two-phase continua near drained and undrained limits, formation of deformation bands and severe seepage or runoff.

\section{Acknowledgments}

We thank the two anonymous reviewers for their helpful suggestions and feedback, and for providing us reference relevant to this research. This research is supported by the Earth Materials and Processes program from the US Army Research Office under grant contract W911NF-18-2-0306, the Dynamic Materials and Interactions Program from the Air Force Office of Scientific Research, USA under grant contract FA9550-17-1-0169, the Nuclear Energy University Program from the Department of Energy, USA under grant contract DE-NE0008534, the NSF CAREER grant from Mechanics of Materials and Structures program at National Science Foundation, USA under grant contract CMMI-1846875 as well as Internal Research Funds (IRF) from Faculty of Engineering at McMaster University, Canada. These supports are gratefully acknowledged. The views and conclusions contained in this document are those of the authors, and should not be interpreted as representing the official policies, either expressed or implied, of the sponsors, including the Army Research Laboratory or the U.S. Government. The U.S. Government is authorized to reproduce and distribute reprints for Government purposes notwithstanding any copyright notation herein.

\section{Appendix A. Stress-point simulations for model calibration}

Material parameter calibration of the local anisotropic Cam-Clay model is conducted using the previous experimental data [95], where three sets of triaxial compression tests were conducted for Tournemire shale 
Table 1

Calibrated plasticity parameters.

\begin{tabular}{lllll}
\hline$\lambda_{\mathrm{p}}$ & $M$ & $\alpha^{\mathrm{p}}$ & $\beta^{\mathrm{p}}$ & $\gamma^{\mathrm{p}}$ \\
\hline 0.003 & 1.0 & 0.784 & 0.565 & 1.000 \\
\hline
\end{tabular}

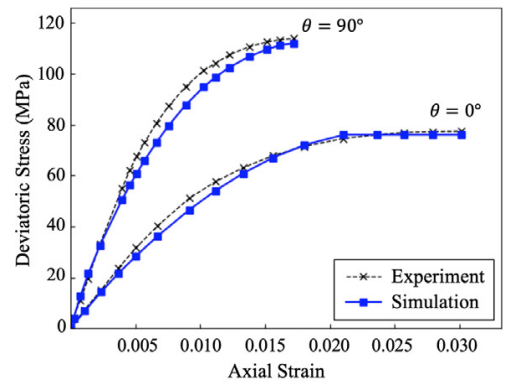

(a) Confining stress $\sigma_{c}=-30 \mathrm{MPa}$

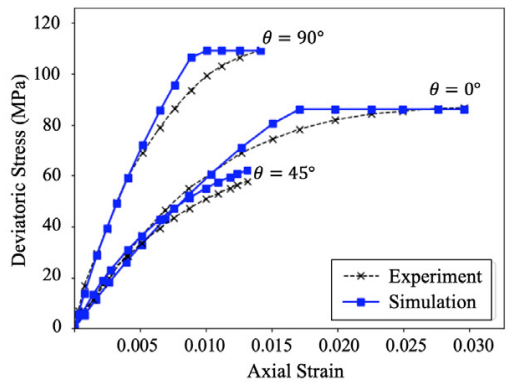

(b) Confining stress $\sigma_{c}=-40 \mathrm{MPa}$

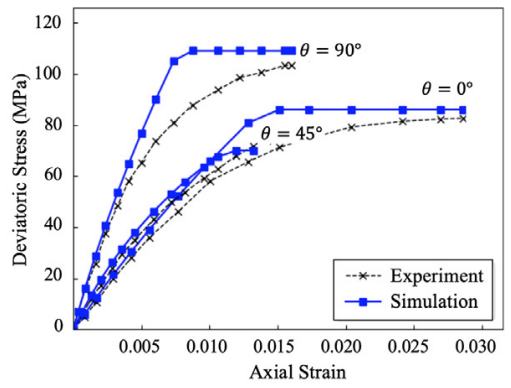

(b) Confining stress $\sigma_{c}=-50 \mathrm{MPa}$

Fig. 15. Comparison of simulation results and experimental data [95] at different confining stresses: (a) $\sigma_{c}^{\prime}=-30 \mathrm{MPa}$; (b) $\sigma_{c}^{\prime}=-40 \mathrm{MPa}$; and (c) $\sigma_{c}^{\prime}=50 \mathrm{MPa}$.

specimens at different confining pressure. In each set of the tests, two to three specimens of different bedding orientations at $\theta=0^{\circ}, 45^{\circ}$, and $90^{\circ}$ are prepared. Similar calibration works were performed by Semnani et al. [22] and Bryant and Sun [98], respectively. The similar procedure is performed here based on the assumption that the experimental specimens are initially homogeneous followed by homogeneous deformation.

The calibration leverages the open source software toolkit Dakota [99], in which the gradient-based least square algorithm (NL2SOL) is used. Third optimization procedure minimizes the objective function which can be varied, depending on the problem of interest. Here the objective function is find one set of material parameters that minimizes the discrepancy between experimental data and simulation results, that is, the deviatoric stress-strain history for specimens of different orientations subjected to triaxial loading.

To simplify the calibration procedure for plasticity material parameters the preliminary simulations are performed to first identify elastic material parameters (cf., [37]). By using only experimental data at $\sigma_{c}^{\prime}=-40 \mathrm{MPa}$ with the axial strain less then 0.005 , this initial calibration yields the elastic material properties as: $E_{1}=20,822 \mathrm{MPa}$, $E_{2}=10,175 \mathrm{MPa}, v_{12}=0.25, v_{23}=0.27$, and $G_{12}=2000 \mathrm{MPa}$ (cf., [22]). Subsequently, an inverse problem is solved to identify one set of the plastic material parameters (with the fixed elasticity parameters listed above) for different confining pressure and bedding orientations.

Fig. 15 shows the best-fit curves of simulations compared to the experiment results. This comparison indicates that the local anisotropic Cam-Clay model well captures the overall behavior of Tournemire shale specimen. The calibrated plastic material parameters $\left(M, \alpha^{\mathrm{p}}, \beta^{\mathrm{p}}, \gamma^{\mathrm{p}}\right)$ are presented in Table 1.

As discussed in the previous work Semnani et al. [22], Bryant and Sun [37], the current anisotropic Cam-Clay model does not fully take into account the initial fracture, crack propagation, or heterogeneity in samples. Readers interested at anisotropic damage originated from faults or bedding structures may refer to work such as Pandolfi et al. [1], Ortiz and Pandolfi [100], Della Vecchia et al. [2], De Bellis et al. [3].

\section{Appendix B. Computational efficiency}

We investigate computational performance of the proposed adaptive meshing approach in terms of the simulation time. In general, the higher accuracy in numerical simulations requires the small size of discretizations. Because the location of interest, such as strain localization, in the domain is not a priori information, the natural approach is to use reasonably small elements over the whole domain, which often demands higher computational calculations. The concept of adaptive meshing can resolve this issue by accommodating the refined mesh around the location of interest while the other domain is remained the coarser mesh. In other words, the adaptive meshing technique can save the computational time by reducing the number of elements but still using small spatial discretizations when needed. 
Table 2

Information of numerical simulations.

\begin{tabular}{lll}
\hline Information & Uniform mesh & Adaptive mesh \\
\hline Initial elements & 3200 & 800 \\
Final elements & 3200 & 1328 \\
Simulation time & $4350(\mathrm{~s})$ & $2180(\mathrm{~s})$ \\
\hline
\end{tabular}

* The same load increment is set for both conditions.

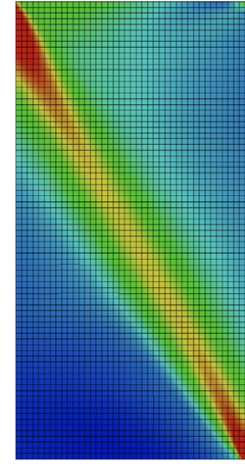

$<$ Uniform Mesh>

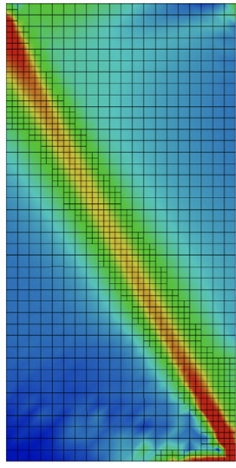

$<$ Adaptive Mesh $>$

(a)

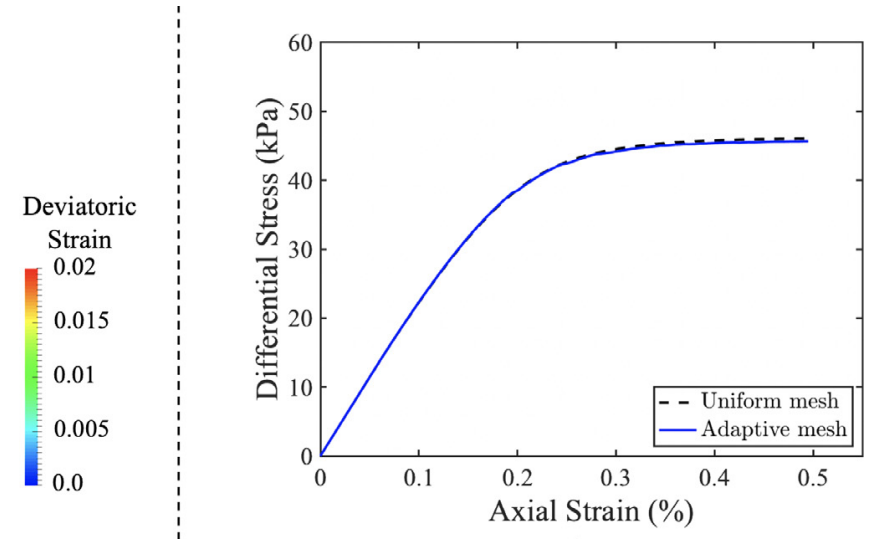

(b)

Fig. 16. Comparison of simulation results with uniform and adaptive meshes: (a) the patterns of deviatoric strain, and (b) the stress-strain curves.

The same boundary value problem is designed to two different mesh conditions: uniform and adaptive meshes. The schematics of loading and boundary conditions for biaxial tests is depicted in Fig. 2. For both cases, we consider the material parameters calibrated from Appendix A with the bedding orientation $\theta=45^{\circ}: E_{1}=20,822 \mathrm{MPa}$, $E_{2}=10,175 \mathrm{MPa}, v_{12}=0.25, v_{23}=0.27, G_{12}=2000, \lambda_{\mathrm{p}}, M=1.0, \alpha^{\mathrm{p}}=0.784, \beta^{\mathrm{p}}=0.565$, and $\gamma^{\mathrm{p}}=1.0$. The plastic length scale and penalty parameters for micromorphic regularization are assumed as, $l_{p}=2.0 \times 10^{-2} \mathrm{~mm}$ and $\varepsilon_{p}=10$, with the mesh refinement criteria $G_{I}=3.0 \times 10^{-4} \mathrm{~N}$.

We then adopt the size of the smallest element as constraints for discretization. This design affects the initial number of finite elements as in Table 2, in which the uniform mesh has 3200 finite elements while the adaptive mesh has the initial and final elements of 800 and 1328, respectively. As expected, the simulation time for the adaptive mesh is reduced about a half compared to that of the uniform mesh. Although the computational time is not significantly diminished in this numerical test, the advantage of using adaptive meshing will be increased as the domain size increases.

Fig. 16 presents the deviatoric strain patterns and the resultant strain-stress curves obtained from both simulations using the uniform and adaptive mesh. These figures further confirm that the proposed adaptive meshing technique yields numerical results consistently while saving the computational time.

\section{Appendix C. Performance of adaptive meshing and micromorphic regularization}

We demonstrate the performance of adaptive meshing techniques associated with micromorphic regularization under the plane strain condition. We first create an isotropic numerical specimen by setting $\boldsymbol{P}^{\mathrm{p}}=\boldsymbol{I}^{4}$ and $\alpha^{\mathrm{p}}=\beta^{\mathrm{p}}=\gamma^{\mathrm{p}}=1$ for control experiments. The boundary conditions and schematics of the numerical domain are depicted in Fig. 2. Note that all boundary surfaces are assumed to be impermeable. The width and height of the specimen are $B=0.05 \mathrm{~m}$ and $L=0.1 \mathrm{~m}$, respectively. Due to the isotropic property of the material, only the right half of the domain is used in the simulation. As such, the horizontal directions are constrained on the left and bottom surfaces of the domain, and the displacement of the top surface is controlled to compress the specimen. The confining pressure is applied on the right surface, and the specimen is globally undrained. In other words, pore fluid may flow within the specimen but there is no flux across the boundary of the domain. We select two uniform 

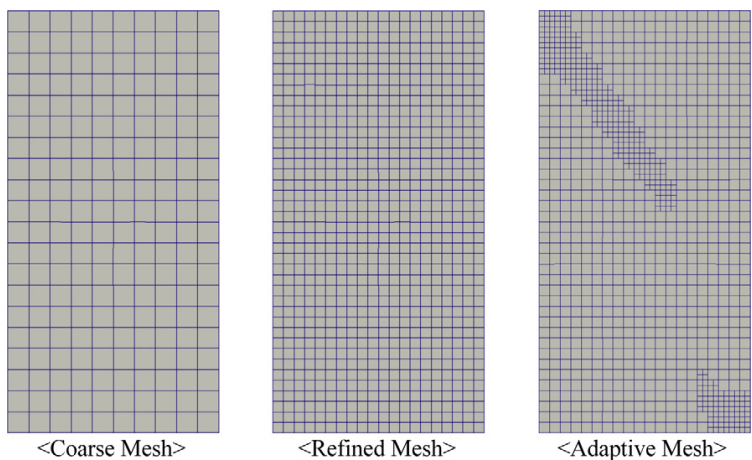

Fig. 17. Description of the two uniform meshes and the adaptive mesh of the biaxial compression problem.

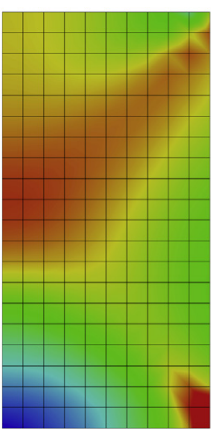

$<$ Coarse Mesh $>$

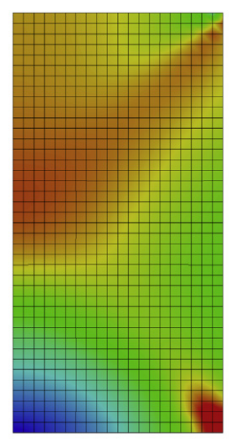

$<$ Refined Mesh>

(a)

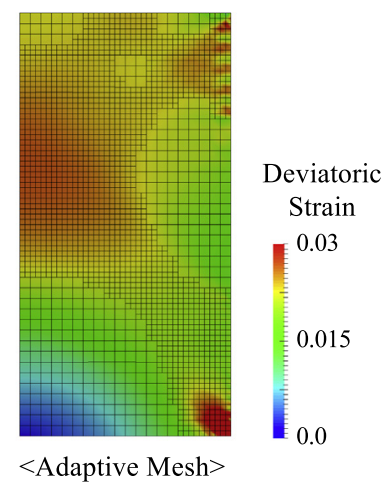

$<$ Adaptive Mesh $>$

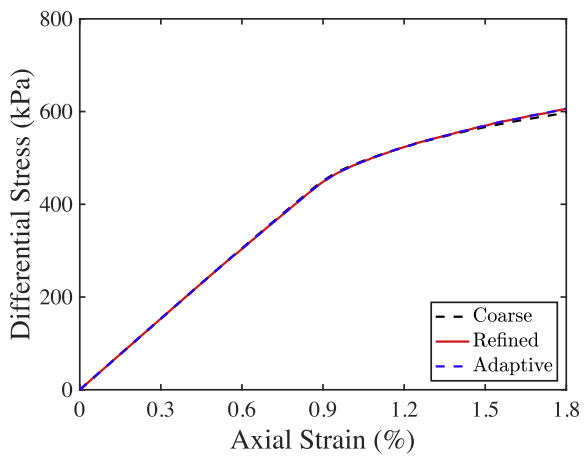

(b)

Fig. 18. Performance of the proposed model with different mesh conditions when the initial OCR is 1.25 - the micromorphic regularization is not introduced: (a) the deviatoric strain patterns under the uniform (coarse and refined) and adaptive mesh conditions; (b) the stress-strain curves.

meshes (denoted as Coarse and Refined) and one adaptive mesh (denoted as Adaptive) as the benchmark cases and compare the simulations results obtained from two uniform meshes with the adaptive mesh results. The adaptive mesh refinement process is activated from the given refined mesh as shown in Fig. 17. Given these meshes, we then conduct numerical simulations of biaxial compression tests conducted with different over-consolidation ratios (1.25 and 4.0) to trigger hardening and softening behaviors of the Cam-Clay materials. Other material parameters for the anisotropic critical state plasticity model are $E=30,000 \mathrm{kPa}, v=0.3, \lambda_{p}=0.08$ and $M=1$.

\section{C.1. Simulations on lightly over-consolidated materials}

In the case of initial $\mathrm{OCR}=1.25$, the global responses are not sensitive to the mesh size regardless of whether micromorphic regularization method is used. This result is expected as the material exhibits associative plastic flow and strain hardening [90,101-105]. Here the initial preconsolidation stress $\left(p_{c_{0}}^{\prime}\right)$ is set to $-1000 \mathrm{kPa}$ while the initial confining pressure $\left(p_{0}^{\prime}\right)$ is $-800 \mathrm{kPa}$. The results of a series of simulations are presented in Fig. 18(a), in which the patterns of deviatoric strain are shown at the end of biaxial compression simulations conducted with different mesh conditions. For adaptive mesh refinement, the refinement is activated in the initial refined uniform mesh with the refinement criteria $G_{c r i t}^{I}=1.0 \times 10^{-2} \mathrm{kN}$. Although no micromorphic regularization is introduced, the simulations of the uniform mesh do not exhibit mesh sensitivity. While the strain is more concentrated in the refined regions, the strain distribution of the numerical specimen discretized by different meshes are very close under the undrained condition. This result is consistent with the homogenized stress-strain curves presented in Fig. 18(b). 


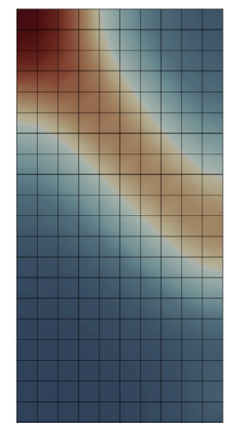

$<$ Coarse Mesh $>$

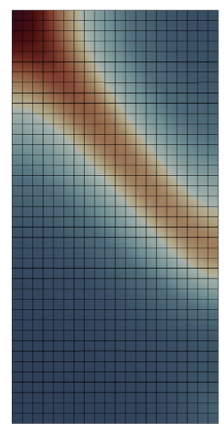

$<$ Refined Mesh $>$

(a)

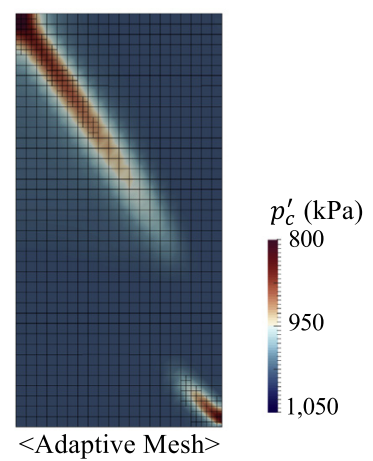

$<$ Adaptive Mesh>

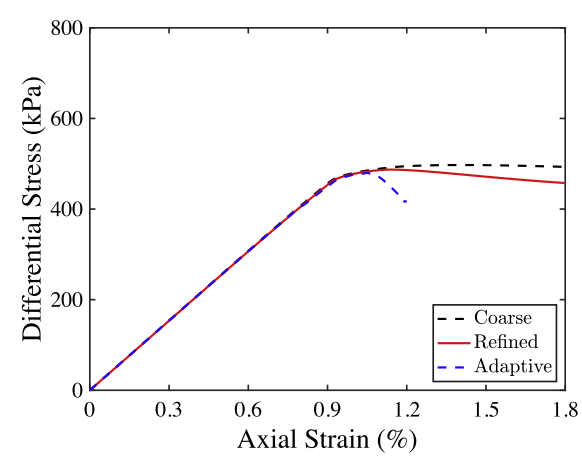

(b)

Fig. 19. No micromorphic regularization when the initial OCR is 4.0 - (a) the patterns of $p_{c}^{\prime}$ to indicate the plastic regions and (b) the stress-strain curves from the three uniform (coarse, medium, and fine) and the adaptive mesh conditions: the more strain localization and softening behaviors as the mesh refined.

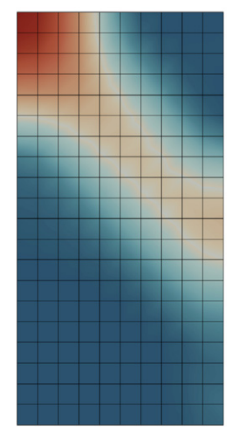

$<$ Coarse Mesh $>$

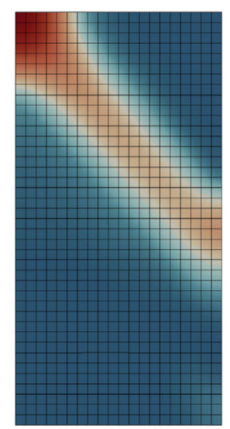

$<$ Refined Mesh $>$

(a)

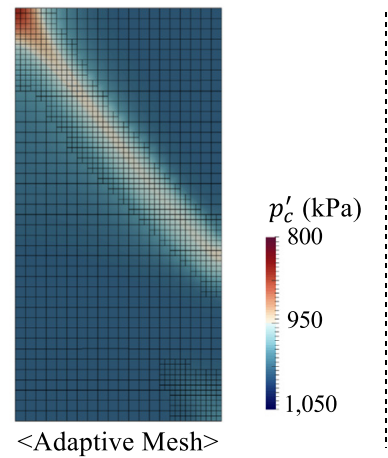

$<$ Adaptive Mesh>

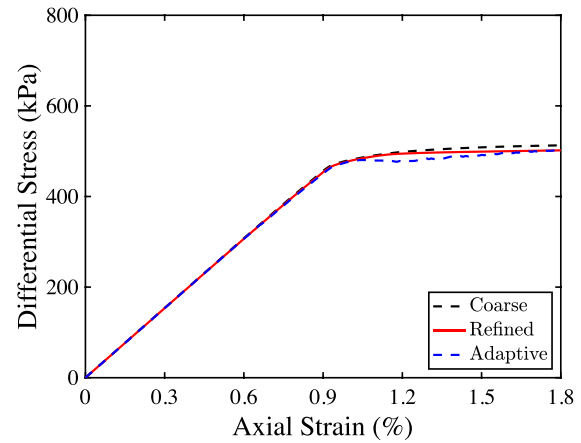

(b)

Fig. 20. Effect of introducing micromorphic regularization when the initial OCR is 4.0 - (a) the patterns of $p_{c}^{\prime}$ to indicate the plastic regions and (b) the regularized stress-strain curves: the regularized patterns of plastic region and consistent stress-strain curves under the different mesh conditions.

\section{C.2. Simulations on heavily over-consolidated materials}

We proceed to illustrate the performance of the proposed model with the initial OCR of 4.0, in which $p_{c_{0}}^{\prime}=$ $-1000 \mathrm{kPa}$ and $p_{0}^{\prime}=-250 \mathrm{kPa}$. Firstly, we observe the mesh dependent behavior with respect to both global stressstrain curves and local $p_{c}^{\prime}$ patterns in Fig. 19(a) and (b) when the micromorphic regularization is not accommodated. While the Cam-Clay type models do not show drastic softening behavior and associated strain localization under the uniform mesh, the lack of regularization is clearly identified as the mesh is refined. When the adaptive mesh is introduced with out any regularization, the strain localization is concentrated around the refined region, which yields loss of ellipticity of the proposed model in the much earlier loading stage. To be specific, the various stress-strain curves are collected in Fig. 19(b), where increasing softening behavior is observed as the mesh size decreases. This mesh sensitivity is further found from the distribution of $p_{c}^{\prime}$, which is consistent with narrowing of the width of the localization zone associated with the mesh refinement. This typical phenomenon is a fundamental issue in finite element analyses with respect to localization of deformation (e.g., [12,106-108]).

We then introduce the micromorphic regularization and illustrate how this treatment yields nonlocal effect in Fig. 20. The parameters for regularization setting are: $l_{p}=2.0 \times 10^{-3} \mathrm{~m}$ and $\varepsilon_{p}=0.25$. As we select $p_{c}^{\prime}$ for the micromorphic hardening variable, its distribution is also presented to indicate the patterns of plastic region. Compared to the previous model (Fig. 19(a)), the $p_{c}^{\prime}$ is less concentrated and diffused associated with the plastic 


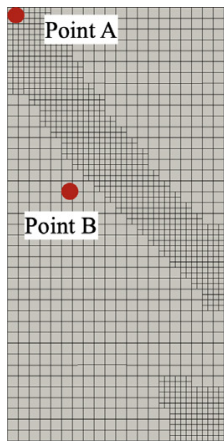

(a)

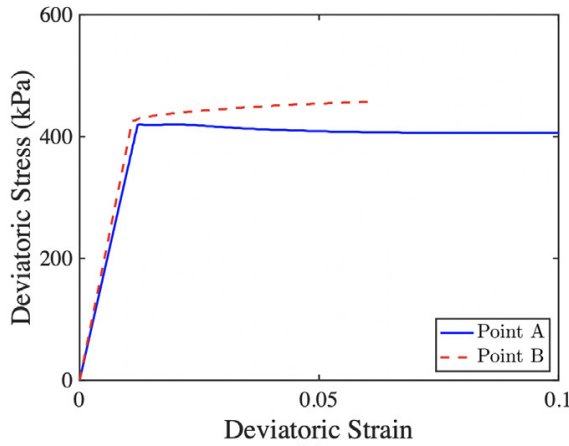

(b)

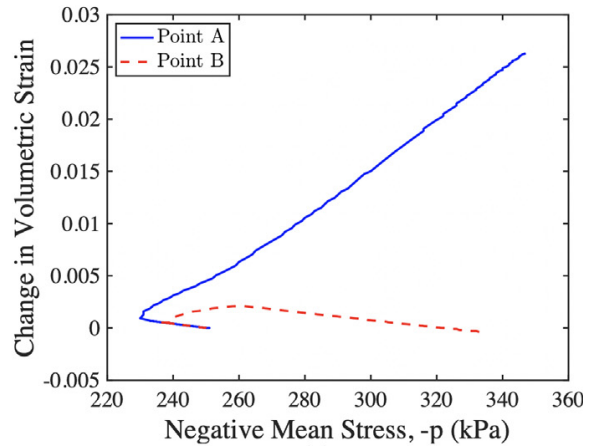

(c)

Fig. 21. Local behavior of two points A (inside the localization region) and B (outside the localization region): (a) locations of Points A and $\mathrm{B}$; (b) stress-strain curves; (c) change in volumetric strain-negative mean pressure $(-p)$ curves.

length scale as the uniform mesh size decreases. This tendency is identified as well when the adaptive mesh refinement is activated. By penalizing the local $(\bar{\alpha})$ and global $(\alpha)$ micromorphic variables, in other words, this approach prevents the strain localization to be isolated into the mesh size. This regularization effect is further observed in Fig. 20(b), where the stress-strain curves from the different mesh conditions indicate consistent mesh-independent behavior. Overall, the proposed model for micromorphic regularization associated with adaptive meshing technique well preserves mesh independence when the initial OCR is 4.0. In the meantime, we note that the micromorphic regularization prevents mesh dependency by diffusing out the local concentration of plastic regions associated with the plastic length scale, which weakens the strain softening (Figs. 19(b) and 20(b)). To further present the local softening under the micromorphic regularization settings, we present local behaviors observed from two different points A and B in Fig. 21(a). Inside the strain localization zone, Point A, the deviatoric stress and volumetric strain change curves clearly show the local softening behavior while the local hardening is observed outside the localization zone at Point B (Fig. 21(b) and (c)).

\section{References}

[1] A. Pandolfi, S. Conti, M. Ortiz, A recursive-faulting model of distributed damage in confined brittle materials, J. Mech. Phys. Solids 54 (9) (2006) 1972-2003.

[2] G. Della Vecchia, M.L. De Bellis, A. Pandolfi, A multiscale microstructural model of damage and permeability in fractured solids, Procedia Eng. 158 (2016) 21-26.

[3] M. De Bellis, G. Della Vecchia, M. Ortiz, A. Pandolfi, A linearized porous brittle damage material model with distributed frictional-cohesive faults, Eng. Geol. 215 (2016) 10-24.

[4] M.L. De Bellis, G. Della Vecchia, M. Ortiz, A. Pandolfi, A multiscale model of distributed fracture and permeability in solids in all-round compression, J. Mech. Phys. Solids 104 (2017) 12-31.

[5] J. Fish, T. Jiang, Z. Yuan, A staggered nonlocal multiscale model for a heterogeneous medium, Internat. J. Numer. Methods Engrg. 91 (2) (2012) 142-157.

[6] J. Hutchinson, N. Fleck, Strain gradient plasticity, in: Advances in Applied Mechanics, Vol. 33, Academic, 1997, pp. $295-361$.

[7] W.D. Nix, H. Gao, Indentation size effects in crystalline materials: a law for strain gradient plasticity, J. Mech. Phys. Solids 46 (3) (1998) 411-425.

[8] H.-B. Mühlhaus, E. Alfantis, A variational principle for gradient plasticity, Int. J. Solids Struct. 28 (7) (1991) $845-857$.

[9] Z.P. Bažant, Scaling of dislocation-based strain-gradient plasticity, J. Mech. Phys. Solids 50 (3) (2002) 435-448.

[10] C. Miehe, S. Teichtmeister, F. Aldakheel, Phase-field modelling of ductile fracture: a variational gradient-extended plasticity-damage theory and its micromorphic regularization, Phil. Trans. R. Soc. A 374 (2066) (2016) 20150170.

[11] K. Wang, W. Sun, S. Salager, S. Na, G. Khaddour, Identifying material parameters for a micro-polar plasticity model via x-ray micro-computed tomographic (ct) images: lessons learned from the curve-fitting exercises, Int. J. Multiscale Comput. Eng. 14 (4) (2016).

[12] R. De Borst, H.-B. Mühlhaus, Gradient-dependent plasticity: Formulation and algorithmic aspects, Internat. J. Numer. Methods Engrg. 35 (3) (1992) 521-539.

[13] H. Gao, Y. Huang, W. Nix, J. Hutchinson, Mechanism-based strain gradient plasticity—i. theory, J. Mech. Phys. Solids 47 (6) (1999) 1239-1263.

[14] Z.P. Bažant, M. Jirásek, Nonlocal integral formulations of plasticity and damage: survey of progress, J. Eng. Mech. 128 (11) (2002) 1119-1149. 
[15] C. Lim, G. Zhang, J. Reddy, A higher-order nonlocal elasticity and strain gradient theory and its applications in wave propagation, J. Mech. Phys. Solids 78 (2015) 298-313.

[16] K. Wang, W. Sun, A unified variational eigen-erosion framework for interacting brittle fractures and compaction bands in fluid-infiltrating porous media, Comput. Methods Appl. Mech. Engrg. 318 (2017) 1-32.

[17] C. Miehe, F. Aldakheel, S. Mauthe, Mixed variational principles and robust finite element implementations of gradient plasticity at small strains, Int. J. Numer. Methods Eng. 94 (11) (2013) 1037-1074.

[18] C. Miehe, F. Aldakheel, S. Teichtmeister, Phase-field modeling of ductile fracture at finite strains: A robust variational-based numerical implementation of a gradient-extended theory by micromorphic regularization, Internat. J. Numer. Methods Engrg. 111 (9) (2017) $816-863$.

[19] F. Aldakheel, Micromorphic approach for gradient-extended thermo-elastic-plastic solids in the logarithmic strain space, Contin. Mech. Thermodyn. (2017) 1-11.

[20] S. Forest, Nonlinear regularization operators as derived from the micromorphic approach to gradient elasticity, viscoplasticity and damage, Proc. R. Soc. Lond. Ser. A Math. Phys. Eng. Sci. 472 (2188) (2016) 20150755.

[21] S. Forest, Micromorphic approach for gradient elasticity, viscoplasticity, and damage, J. Eng. Mech. 135 (3) (2009) $117-131$.

[22] S.J. Semnani, J.A. White, R.I. Borja, Thermoplasticity and strain localization in transversely isotropic materials based on anisotropic critical state plasticity, Int. J. Numer. Anal. Methods Geomech. 40 (18) (2016) 2423-2449.

[23] A. Papastavrou, P. Steinmann, On deformational and configurational poro-mechanics: dissipative versus non-dissipative modelling of two-phase solid/fluid mixtures, Arch. Appl. Mech. 80 (9) (2010) 969-984.

[24] F. Aldakheel, C. Miehe, Coupled thermomechanical response of gradient plasticity, Int. J. Plast. 91 (2017) 1-24.

[25] K. Roscoe, J. Burland, On the generalized stress-strain behaviour of wet clay, in: Engineering Plasticity, Cambridge University Press, 1968.

[26] D. Versino, K.C. Bennett, Generalized radial-return mapping algorithm for anisotropic von mises plasticity framed in material eigenspace, Internat. J. Numer. Methods Engrg. 116 (3) (2018) 202-222.

[27] R.I. Borja, Plasticity: Modeling \& Computation, Springer Science \& Business Media, 2013.

[28] R. Borja, C. Tamagnini, Cam-clay plasticity part iii: Extension of the infinitesimal model to include finite strains, Comput. Methods Appl. Mech. Engrg. 155 (1) (1998) 73-95.

[29] S. Na, W. Sun, Computational thermo-hydro-mechanics for multiphase freezing and thawing porous media in the finite deformation range, Comput. Methods Appl. Mech. Engrg. 318 (2017) 667-700.

[30] J.A. White, R.I. Borja, Stabilized low-order finite elements for coupled solid-deformation/fluid-diffusion and their application to fault zone transients, Comput. Methods Appl. Mech. Engrg. 197 (49-50) (2008) 4353-4366.

[31] R.I. Borja, C. Tamagnini, A. Amorosi, Coupling plasticity and energy-conserving elasticity models for clays, J. Geotech. Geoenviron. Eng. 123 (10) (1997) 948-957.

[32] R.A. Engelen, M.G. Geers, F.P. Baaijens, Nonlocal implicit gradient-enhanced elasto-plasticity for the modelling of softening behaviour, Int. J. Plast. 19 (4) (2003) 403-433.

[33] M. Geers, R. Peerlings, W. Brekelmans, R. de Borst, Phenomenological nonlocal approaches based on implicit gradient-enhanced damage, Acta Mech. 144 (1-2) (2000) 1-15.

[34] R. Peerlings, M. Geers, R. De Borst, W. Brekelmans, A critical comparison of nonlocal and gradient-enhanced softening continua, Int. J. Solids Struct. 38 (44-45) (2001) 7723-7746.

[35] R. Peerlings, On the role of moving elastic-plastic boundaries in strain gradient plasticity, Modelling Simulation Mater. Sci. Eng. 15 (1) (2006) S109.

[36] K. Wang, W. Sun, A semi-implicit micropolar discrete-to-continuum method for granular materials, in: M. Papadrakakis, V. Papadopoulos, G. Stefanou, V. Plevris (Eds.), Proceedings of European Congress on Computational Methods in Applied Science and Engineering, Crete Island, 2016, pp. 5-10.

[37] E.C. Bryant, W. Sun, A mixed-mode phase field fracture model in anisotropic rocks with consistent kinematics, Comput. Methods Appl. Mech. Engrg. 342 (2018) 561-584.

[38] A. Qinami, E.C. Bryant, W. Sun, M. Kaliske, Circumventing mesh bias by r-and h-adaptive techniques for variational eigenfracture, Int. J. Fract. (2019) 1-14.

[39] A.R. Díaz, N. Kikuchi, J.E. Taylor, A method of grid optimization for finite element methods, Comput. Methods Appl. Mech. Engrg. 41 (1) (1983) 29-45.

[40] A. Needleman, Material rate dependence and mesh sensitivity in localization problems, Comput. Methods Appl. Mech. Engrg. 67 (1) (1988) 69-85.

[41] M. Ortiz, J. Quigley IV, Adaptive mesh refinement in strain localization problems, Comput. Methods Appl. Mech. Engrg. 90 (1-3) (1991) 781-804.

[42] R. Verfürth, A posteriori error estimation and adaptive mesh-refinement techniques, J. Comput. Appl. Math. 50 (1-3) (1994) 67-83.

[43] B.A. Schrefler, S. Secchi, L. Simoni, On adaptive refinement techniques in multi-field problems including cohesive fracture, Comput. Methods Appl. Mech. Engrg. 195 (4-6) (2006) 444-461.

[44] I. Babuvška, W.C. Rheinboldt, Error estimates for adaptive finite element computations, SIAM J. Numer. Anal. 15 (4) (1978) $736-754$.

[45] O.C. Zienkiewicz, J.Z. Zhu, A simple error estimator and adaptive procedure for practical engineerng analysis, Internat. J. Numer. Methods Engrg. 24 (2) (1987) 337-357.

[46] O. Zienkiewicz, J. Zhu, Adaptivity and mesh generation, Internat. J. Numer. Methods Engrg. 32 (4) (1991) $783-810$.

[47] O.C. Zienkiewicz, J.Z. Zhu, The superconvergent patch recovery and a posteriori error estimates. part 1: The recovery technique, Internat. J. Numer. Methods Engrg. 33 (7) (1992) 1331-1364. 
[48] N.S. Lee, K.J. Bathe, Error indicators and adaptive remeshing in large deformation finite element analysis, Finite Elem. Anal. Des. 16 (2) (1994) 99-139.

[49] M. Ainsworth, J.T. Oden, A posteriori error estimation in finite element analysis, Comput. Methods Appl. Mech. Engrg. 142 (1-2) (1997) 1-88.

[50] A. Tabarraei, N. Sukumar, Adaptive computations using material forces and residual-based error estimators on quadtree meshes, Comput. Methods Appl. Mech. Engrg. 196 (25-28) (2007) 2657-2680.

[51] J. Mosler, M. Ortiz, Variational h-adaption in finite deformation elasticity and plasticity, Internat. J. Numer. Methods Engrg. 72 (5) (2007) 505-523.

[52] J. Mosler, M. Ortiz, An error-estimate-free and remapping-free variational mesh refinement and coarsening method for dissipative solids at finite strains, Internat. J. Numer. Methods Engrg. 77 (3) (2009) 437-450.

[53] R. Mueller, G. Maugin, On material forces and finite element discretizations, Comput. Mech. 29 (1) (2002) 52-60.

[54] P. Heintz, F. Larsson, P. Hansbo, K. Runesson, Adaptive strategies and error control for computing material forces in fracture mechanics, Int. J. Numer. Methods Eng. 60 (7) (2004) 1287-1299.

[55] R. Mueller, D. Gross, G. Maugin, Use of material forces in adaptive finite element methods, Comput. Mech. 33 (6) (2004) $421-434$.

[56] M.E. Gurtin, Configurational Forces as Basic Concepts of Continuum Physics, Vol. 137, Springer Science \& Business Media, 2008.

[57] G.A. Maugin, Material forces: concepts and applications, Appl. Mech. Rev. 48 (5) (1995) 213-245.

[58] P. Steinmann, M. Scherer, R. Denzer, Secret and joy of configurational mechanics: From foundations in continuum mechanics to applications in computational mechanics, ZAMM Z. Angew. Math. Mech. 89 (8) (2009) 614-630.

[59] H. Buggisch, D. Gross, K.-H. Krüger, Einige erhaltungssätze der kontinuumsmechanik vom j-integral-typ, Ing.-Arch. 50 (2) (1981) 103-111.

[60] B. Näser, M. Kaliske, R. Müller, Material forces for inelastic models at large strains: application to fracture mechanics, Comput. Mech. 40 (6) (2007) 1005-1013.

[61] G.A. Maugin, Configurational Forces: Thermomechanics, Physics, Mathematics, and Numerics, Chapman and Hall/CRC, 2016.

[62] O. Coussy, J.-M. Pereira, J. Vaunat, Revisiting the thermodynamics of hardening plasticity for unsaturated soils, Comput. Geotech. 37 (1-2) (2010) 207-215.

[63] W. Sun, J.E. Andrade, Diffuse bifurcations of porous media under partially drained conditions, in: Multiscale and Multiphysics Processes in Geomechanics, Springer, 2011, pp. 61-64.

[64] W. Sun, Z. Cai, J. Choo, Mixed arlequin method for multiscale poromechanics problems, Internat. J. Numer. Methods Engrg. 111 (7) (2017) 624-659.

[65] L. Walpole, Fourth-rank tensors of the thirty-two crystal classes: multiplication tables, Proc. R. Soc. Lond. Ser. A Math. Phys. Eng. Sci. 391 (1800) (1984) 149-179.

[66] A.J. Crook, J.-G. Yu, S.M. Willson, et al., Development of an orthotropic 3d elastoplastic material model for shale, in: SPE/ISRM Rock Mechanics Conference, Society of Petroleum Engineers, 2002.

[67] M. Ortiz, L. Stainier, The variational formulation of viscoplastic constitutive updates, Comput. Methods Appl. Mech. Engrg. 171 (3-4) (1999) 419-444.

[68] K. Weinberg, A. Mota, M. Ortiz, A variational constitutive model for porous metal plasticity, Comput. Mech. 37 (2) (2006) $142-152$.

[69] J. Mosler, O. Bruhns, On the implementation of rate-independent standard dissipative solids at finite strain-variational constitutive updates, Comput. Methods Appl. Mech. Engrg. 199 (9-12) (2010) 417-429.

[70] F. Armero, C. Callari, An analysis of strong discontinuities in a saturated poro-plastic solid, Internat. J. Numer. Methods Engrg. 46 (10) (1999) 1673-1698.

[71] C. Miehe, S. Mauthe, Phase field modeling of fracture in multi-physics problems. part iii. crack driving forces in hydro-poro-elasticity and hydraulic fracturing of fluid-saturated porous media, Comput. Methods Appl. Mech. Engrg. 304 (2016) 619-655.

[72] D.J. Payen, K.-J. Bathe, The use of nodal point forces to improve element stresses, Comput. Struct. 89 (5-6) (2011) $485-495$.

[73] A. Mota, W. Sun, J.T. Ostien, J.W. Foulk, K.N. Long, Lie-group interpolation and variational recovery for internal variables, Comput. Mech. (2013) 1-19.

[74] T.J. Truster, O. Nassif, Variational projection methods for gradient crystal plasticity using lie algebras, Internat. J. Numer. Methods Engrg. 110 (4) (2017) 303-332.

[75] F.C. Park, B. Ravani, Smooth invariant interpolation of rotations, ACM Trans. Graph. 16 (3) (1997) $277-295$.

[76] H. Cramer, M. Rudolph, G. Steinl, W. Wunderlich, A hierarchical adaptive finite element strategy for elastic-plastic problems, Comput. Struct. 73 (1-5) (1999) 61-72.

[77] W. Bangerth, R. Hartmann, G. Kanschat, Deal. ii-a general-purpose object-oriented finite element library, ACM Trans. Math. Software (TOMS) 33 (4) (2007) 24.

[78] W. Bangerth, D. Davydov, T. Heister, L. Heltai, G. Kanschat, M. Kronbichler, M. Maier, B. Turcksin, D. Wells, The deal. ii library, version 8.4, J. Numer. Math. 24 (3) (2016) 135-141.

[79] M.A. Biot, General theory of three-dimensional consolidation, J. Appl. Phys. 12 (2) (1941) 155-164.

[80] M.A. Biot, Theory of elasticity and consolidation for a porous anisotropic solid, J. Appl. Phys. 26 (2) (1955) $182-185$.

[81] R.I. Borja, One-step and linear multistep methods for nonlinear consolidation, Comput. Methods Appl. Mech. Engrg. 85 (3) (1991) 239-272.

[82] W. Sun, A stabilized finite element formulation for monolithic thermo-hydro-mechanical simulations at finite strain, Internat. J. Numer. Methods Engrg. 103 (11) (2015) 798-839.

[83] J. Choo, R. Borja, Stabilized mixed finite elements for deformable porous media with double porosity, Comput. Methods Appl. Mech. Engrg. 293 (2015) 131-154. 
[84] W. Sun, J.T. Ostien, A.G. Salinger, A stabilized assumed deformation gradient finite element formulation for strongly coupled poromechanical simulations at finite strain, Int. J. Numer. Anal. Methods Geomech. 37 (16) (2013) 2755-2788.

[85] O.C. Zienkiewicz, A. Chan, M. Pastor, B. Schrefler, T. Shiomi, Computational Geomechanics, Wiley Chichester, 1999.

[86] M. Preisig, J.H. Prévost, Stabilization procedures in coupled poromechanics problems: A critical assessment, Int. J. Numer. Anal. Methods Geomech. 35 (11) (2011) 1207-1225.

[87] W. Sun, Q. Chen, J.T. Ostien, Modeling the hydro-mechanical responses of strip and circular punch loadings on water-saturated collapsible geomaterials, Acta Geotech. (2013).

[88] K. Wang, W. Sun, A semi-implicit discrete-continuum coupling method for porous media based on the effective stress principle at finite strain, Comput. Methods Appl. Mech. Engrg. 304 (2016) 546-583.

[89] J. Kim, H.A. Tchelepi, R. Juanes, et al., Stability, accuracy and efficiency of sequential methods for coupled flow and geomechanics, in: SPE Reservoir Simulation Symposium, Society of Petroleum Engineers, 2009.

[90] K. Wang, W. Sun, A semi-implicit discrete-continuum coupling method for porous media based on the effective stress principle at finite strain, Comput. Methods Appl. Mech. Engrg. 304 (2016) 546-583.

[91] S. Na, W. Sun, Computational thermomechanics of crystalline rock, part i: A combined multi-phase-field/crystal plasticity approach for single crystal simulations, Comput. Methods Appl. Mech. Engrg. 338 (2018) 657-691.

[92] M. Ambati, T. Gerasimov, L. De Lorenzis, A review on phase-field models of brittle fracture and a new fast hybrid formulation, Comput. Mech. 55 (2) (2015) 383-405.

[93] J. Choo, W. Sun, Coupled phase-field and plasticity modeling of geological materials: from brittle fracture to ductile flow, Comput. Methods Appl. Mech. Engrg. 330 (2018) 1-32.

[94] M.J. Borden, T.J. Hughes, C.M. Landis, A. Anvari, I.J. Lee, A phase-field formulation for fracture in ductile materials: Finite deformation balance law derivation, plastic degradation, and stress triaxiality effects, Comput. Methods Appl. Mech. Engrg. 312 (2016) 130-166.

[95] H. Niandou, J. Shao, J. Henry, D. Fourmaintraux, Laboratory investigation of the mechanical behaviour of tournemire shale, Int. J. Rock Mech. Min. Sci. 34 (1) (1997) 3-16.

[96] Y. Zhao, S.J. Semnani, Q. Yin, R.I. Borja, On the strength of transversely isotropic rocks, Int. J. Numer. Anal. Methods Geomech. 42 (16) (2018) 1917-1934.

[97] Y.M. Tien, M.C. Kuo, C.H. Juang, An experimental investigation of the failure mechanism of simulated transversely isotropic rocks, Int. J. Rock Mech. Min. Sci. 43 (8) (2006) 1163-1181.

[98] E.C. Bryant, W. Sun, A micromorphically regularized Cam-clay model for capturing size-dependent anisotropy of geomaterials, Comput. Methods Appl. Mech. Engrg. 354 (2019) 56-95.

[99] B.M. Adams, W. Bohnhoff, K. Dalbey, J. Eddy, M. Eldred, D. Gay, K. Haskell, P.D. Hough, L. Swiler, Dakota, a multilevel parallel object-oriented framework for design optimization, parameter estimation, uncertainty quantification, and sensitivity analysis: version 5.0 user's manual. Sandia National Laboratories, Tech. Rep. SAND2010-2183, 2009.

[100] M. Ortiz, A. Pandolfi, A variational cam-clay theory of plasticity, Comput. Methods Appl. Mech. Engrg. 193 (27-29) (2004) 2645-2666.

[101] J.W. Rudnicki, J. Rice, Conditions for the localization of deformation in pressure-sensitive dilatant materials, J. Mech. Phys. Solids 23 (6) (1975) 371-394.

[102] W. Sun, A unified method to predict diffuse and localized instabilities in sands, Geomech. Geoeng. 8 (2) (2013) 65-75.

[103] Y. Liu, W. Sun, Z. Yuan, J. Fish, A nonlocal multiscale discrete-continuum model for predicting mechanical behavior of granular materials, Internat. J. Numer. Methods Engrg. 106 (2) (2016) 129-160.

[104] K. Wang, W. Sun, Meta-modeling game for deriving theory-consistent, microstructure-based traction-separation laws via deep reinforcement learning, Comput. Methods Appl. Mech. Engrg. 346 (2019) 216-241.

[105] K. Wang, W. Sun, Q. Du, A cooperative game for automated learning of elasto-plasticity knowledge graphs and models with ai-guided experimentation, Comput. Mech. (2019) 1-33.

[106] R. De Borst, L. Sluys, H.-B. Muhlhaus, J. Pamin, Fundamental issues in finite element analyses of localization of deformation, Eng. Comput. 10 (2) (1993) 99-121.

[107] A. Eringen, On nonlocal plasticity, Internat. J. Engrg. Sci. 19 (12) (1981) 1461-1474.

[108] I. Vardoulakis, E. Aifantis, A gradient flow theory of plasticity for granular materials, Acta Mech. 87 (3-4) (1991) $197-217$. 